\title{
Heterotic/heterotic and heterotic/F-theory duality
}

\author{
Lara B. Anderson, ${ }^{1,2, *}$ He Feng, ${ }^{1, \dagger}$ Xin Gao, ${ }^{3,4, \pitchfork}$ and Mohsen Karkheiran ${ }^{1,2, \S}$ \\ ${ }^{1}$ Department of Physics, Robeson Hall, Virginia Tech, Blacksburg, Virginia 24061, USA \\ ${ }^{2}$ Simons Center for Geometry and Physics, Stony Brook, New York 11794, USA \\ ${ }^{3}$ Dipartimento di Fisica, Universita di Roma "Tor Vergata", Rome 00133, Italy \\ ${ }^{4}$ INFN Sezione di Roma "Tor Vergata”, Rome 00133, Italy
}

(Received 23 July 2019; published 12 December 2019)

\begin{abstract}
We consider heterotic target space dual $(0,2)$ gauged linear sigma models on elliptically fibered CalabiYau manifolds. In this context, each half of the "dual" heterotic theories must in turn have an F-theory dual. Moreover, the apparent relationship between two heterotic compactifications seen in $(0,2)$ heterotic target space dual pairs should, in principle, induce some putative correspondence between the dual F-theory geometries. It has previously been conjectured in the literature that $(0,2)$ target space duality might manifest in F-theory as multiple $K 3$ fibrations of the same elliptically fibered Calabi-Yau manifold. We investigate this conjecture in the context of both six-dimensional and four-dimensional effective theories and demonstrate that, in general, $(0,2)$ target space duality cannot be explained by such a simple phenomenon alone. In all cases, we provide evidence that nongeometric data in F-theory must play at least some role in the induced F-theory correspondence while leaving the full determination of the putative new F-theory duality to future work.
\end{abstract}

DOI: 10.1103/PhysRevD.100.126014

\section{INTRODUCTION}

Heterotic target space duality was first observed in [1] and further explored in [2-6]. The basic premise is simple to state: Two distinct $(0,2)$ gauged linear sigma model (GLSMs) sharing a nongeometric (i.e., Landau-Ginzburg or hybrid) phase can be found to have apparently identical four-dimensional, $\mathcal{N}=1$ target space theories. In these cases, the GLSMs are distinct and the geometric phases of the two theories lead to manifestly different Calabi-Yau manifolds and vector bundles over them. However, the ensuing four-dimensional theories, arising as large volume compactifications of the $E_{8} \times E_{8}$ heterotic string, contain at least the same gauge symmetry and four-dimensional massless particle spectrum. Although not yet understood as a true string duality, this phenomenon has been referred to as $(0,2)$ target space duality (TSD) [1]. A more recent "landscape" survey of such theories $[3,6]$ showed that it is not just in special cases that such dualities can occur; rather it is the vast majority of $(0,2)$ GLSMs containing

\footnotetext{
*lara.anderson@vt.edu

†enghe@vt.edu

*xingao@roma2.infn.it

§mohsenka@vt.edu
}

Published by the American Physical Society under the terms of the Creative Commons Attribution 4.0 International license. Further distribution of this work must maintain attribution to the author(s) and the published article's title, journal citation, and DOI. Funded by SCOAP. nongeometric phases that can be linked to other $(0,2)$ GLSMs in this way. Moreover, recent work [2] demonstrated that in some cases TSD also seems to preserve the form of nontrivial D- and F-term potentials of the fourdimensional theory to a remarkable degree.

In this work, we aim to further explore the consequences of target space duality in the context of yet another duality - that between heterotic string compactifications and F-theory. As has been observed since the first investigations into TSD $[4,7]$, this nontrivial duality of distinct heterotic backgrounds could potentially also lead to an entirely new duality structure within F-theory. Since heterotic and F-theory vacua consist of two of the most promising frameworks for string model building within four-dimensional string compactifications, it make sense to search for such novel and unexplored dualities to better understand redundancies within the space of such theories. In addition, if new dualities exist, they could also provide deep insight into the structure of the effective physics, or perhaps even new computational tools (as has manifestly proved to be the case with mirror symmetry in type II compactifications of string theory; see, e.g., [8]).

Compactifications of the heterotic string and F-theory can lead to identical effective theories in the situation in which the background geometries of the two theories both exhibit fibration structures [9]. Namely, heterotic string theory compactified on a Calabi-Yau $n$-fold with an elliptic fibration

$$
\pi_{h}: X_{n} \stackrel{\mathbb{E}}{\rightarrow} B_{n-1}
$$


over a base manifold $B_{n-1}$ leads to the same effective physics as F-theory compactified on a Calabi-Yau $(n+1)$ fold with a $K 3$ fibration over the same base manifold, $B_{n-1}$ :

$$
\pi_{f}: Y_{n+1} \stackrel{K 3}{\rightarrow} B_{n-1} .
$$

In order to have a well-defined F-theory background, the $(n+1)$-fold $Y_{n+1}$ must also be elliptically fibered, with compatible elliptic/K3 fibrations $[9,10]$.

In the context of (potential) heterotic/heterotic dualities and heterotic/F-theory duality then, there are a number of natural questions that arise. Suppose that $\left(X_{3}, \pi: V \rightarrow X_{3}\right)$ and $\left(\tilde{X}_{3}, \pi: \tilde{V} \rightarrow \tilde{X}_{3}\right)$ are the requisite background geometries (i.e., manifolds, vector bundles) defining two TSD heterotic theories. Then these questions include the following.

(a) Can target space dual pairs be found in which both $X$ and $\tilde{X}$ are elliptically fibered as in Eq. (1.1)? In principle, these two fibrations need not be related in any obvious way, for example, two topologically distinct Calabi-Yau (CY) threefolds $\pi: X_{3} \rightarrow B_{2}$ and $\tilde{\pi}: \tilde{X}_{3} \rightarrow \tilde{B}_{2}$, with distinct (complex) two-dimensional base manifolds $B_{2}, \tilde{B}_{2}$ to their fibrations.

(b) If such elliptically fibered CY threefold geometries can be found within a TSD pair, this will, in principle, lead to two CY fourfolds, $Y_{4}$ and $\tilde{Y}_{4}$, as dual backgrounds for F-theory. It should follow by construction that these two geometries lead to the same fourdimensional effective theory (or at least the same massless spectrum). How can this apparent duality be understood in the context of F-theory? How are $Y_{4}$ and $\tilde{Y}_{4}$ related?

For the first point, to our knowledge, no explicit pairs of elliptically fibered TSD heterotic geometries have yet appeared in the literature. However, at least one proposal for the latter point has been posited. In [3], it was proposed that if fibered heterotic TSD pairs could be found, one possibility for the induced duality in F-theory would be the existence of a $\mathrm{CY}$ fourfold with a single elliptic fibration but more than one K3 fibration,

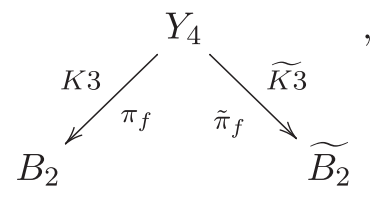

where each fibration can be seen as the F-theory dual of one of the heterotic vacua [associated with $(X, V)$ or $(\tilde{X}, \tilde{V})$, respectively]. Since, by its very definition, F-theory requires that $Y_{4}$ is also elliptically fibered, this would imply that each $K 3$ fiber appearing above is itself also elliptically fibered. Moreover, since the elliptic fibration of F-theory that determines the effective physics (i.e., gauge symmetry, matter spectrum, etc.), in order for the two $K 3$ fibrations to lead to identical effective theories, it would be expected that in fact in this scenario, $Y_{4}$ has only one elliptic fibration, but that it is compatible with two distinct $K 3$ fibrations. If these compatible fibration structures were to exist, it must be that the base of the elliptic fibration, $\rho: Y_{4} \rightarrow B_{3}$, must have two different $\mathbb{P}^{1}$ fibrations:

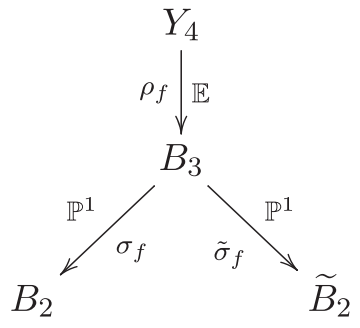

The scenario above is one obvious way in which a "duality" of sorts could arise in F-theory. Of course, in this case, the essential F-theory geometry is not changing, only the $K 3$ fibrations which determine the heterotic dual. This is clearly not the only possibility. As one alternative, it could prove that the F-theory duals of heterotic TSD pairs are in fact two distinct CY fourfolds, $Y_{4}$ and $\tilde{Y}_{4}$, whose gauge symmetries, massless spectra, and effective $\mathcal{N}=1$ potentials are ultimately the same through nontrivial G-flux in the background geometry. We can summarize these two options for the induced duality in F-theory as follows.

(1) (Possibility 1) Heterotic TSD $\Leftrightarrow$ multiple $K 3$ fibrations in a single F-theory geometry (and hence manifestly leading to the same effective physics).

(2) (Possibility 2) Heterotic TSD $\Leftrightarrow$ two distinct pairs of manifolds and G-flux, $\left(Y_{4}, G_{4}\right)$ and $\left(\tilde{Y}_{4}, \tilde{G}_{4}\right)$, which lead to the same effective physics in F-theory.

In this work, we investigate the two questions listed above and provide explicit examples of heterotic target space dual pairs with the requisite fibration structures to lead to F-theory dual theories. As we will outline in the following sections, substantial technical difficulties arise in explicitly computing the full F-theory duals of these heterotic theories. In this work, we do not attempt to fully determine these dual F-theories and instead provide evidence for our primary conclusion: Multiple fibrations in $F$-theory cannot, in general, explain the dual physics of $(0,2) T S D$.

To overcome some of the technical obstacles of heterotic/ F-theory duality, we begin our analysis by actually considering heterotic/F-theory dual pairs in six-dimensional effective theories rather than in four dimensions. In this context, the heterotic duality is a trivial one-TSD pairs simply generate two bundles over $K 3$ with the same second Chern class and are thus trivially guaranteed to give rise to the same massless spectrum (see, e.g., [11]). However, this very simple framework for heterotic TSD pairs allows us to explicitly perform Fourier-Mukai transforms to render the 
data of a holomorphic, stable vector bundle over $K 3$ into its spectral cover [12]. With this data, we are able to explicitly construct examples of F-theory duals and verify that in fact they cannot arise as multiple $K 3$ fibrations of a $\mathrm{CY}$ threefold, $Y_{3}$, determining an F-theory background. The results of this study are presented in Sec. V.

Turning once more to our primary area of interest in $\mathcal{N}=1$ and heterotic compactifications on CY threefolds, we outline the essential ingredients determining the dual F-theory geometry. We find that, in general, a number of technical tools are missing for fully determining the Ftheory physics. Some of these we have developed and will appear separately $[13,14]$, while others we leave to future work. However, we are able to indicate that, in general, the intermediate Jacobians of the dual F-theory geometries must play some role in the new "F-theory duality", whatever it may prove to be. This leads to the presence of essential data associated not with the complex structure of the CY fourfold alone, but with G-flux as well. In the singular limit, such fluxes are well known to have the potential to dramatically change the effective physics through so-called T-brane solutions [15-17] and other possibilities.

In the following sections, we will explore these ideas in detail. The paper is organized in the following way. In Sec. II, we review briefly the essential aspects of $(0,2)$ target space duality. In Sec. III, we provide the first nontrivial examples to appear in the literature of heterotic TSD pairs in which both CY threefolds, $X$ and $\tilde{X}$, are elliptically fibered. In these cases, the heterotic geometries are smooth [consisting of smooth so-called complete intersection Calabi-Yau (CICY) threefolds [18] and stable, holomorphic vector bundles defined via the monad construction [19] over them] and lead to well controlled, perturbative heterotic theories. However, we will demonstrate in this and subsequent sections that existing techniques in the literature to determine dual F-theory geometries, as outlined in Sec. VI, are insufficient to determine the geometry of $Y_{4}$ and $\tilde{Y}_{4}$ in these cases. However, we nonetheless still find some evidence indicating that multiple fibrations of $Y_{4}$ cannot be the F-theory manifestation of $(0,2)$ TSD.

To make concrete the dual F-theory geometry, we move to six-dimensional examples in Sec. V. More precisely, we consider heterotic TSD theories consisting of pairs of bundles over $K 3$ in which the second Chern class of both $V$ and $\tilde{V}$ is taken to be 12 . In this case, it is possible that the F-theory geometry $Y_{3}$ is multiply fibered as described above. However, after finding the spectral data (i.e., the Fourier-Mukai transform) of these bundles, we can explicitly construct the dual F-theory geometry and find that it does not, in general, agree with what can be obtained by multiple fibrations. We will argue further that the F-theory "image" of target space duality under the
heterotic/F-theory map should not be purely geometric, even in six dimensions, but rather it can be related to the intermediate Jacobian of the CY threefold. With these tools and observations in hand, we return to the F-theory duals of four-dimensional, $\mathcal{N}=1$ heterotic theories in Sec. VI.

Finally, in the Appendixes, we consider a handful of examples illustrating both the range of possibilities arising in heterotic TSD dual geometries and potential pitfalls that can arise in constructing dual pairs.

\section{A BRIEF REVIEW OF (0,2) TARGET SPACE DUALITY}

Heterotic target space duality is best understood in the context of heterotic string compactifications associated with $(0,2)$ GLSMs. It was first observed by Distler and Kachru in 1995 [1], and further studied by Blumenhagen [4,5] with a later landscape study [3]. The GLSM provides a description of the complexified compact stringy Kähler moduli space which is divided into various phases [20]. The freedom to vary a Fayet-Iliopolos parameter links a variety of distinct phases including the geometric phases (associated with target space geometries like Calabi-Yau threefolds $X$ and holomorphic vector bundles $V$ ), nongeometric phase (commonly a Landau-Ginzburg phase), and a rich variety of hybrid phases. Described in the $(0,2)$ GLSM language, target space duality is realized by exchanging two certain types of charges in theory, which is defined by $(X, V)$ in the geometric phase, to give a different configuration $(\tilde{X}, \tilde{V})$ from the original one while leaving the superpotential invariant and sharing a common LandauGinzburg phase. Meanwhile, in the geometric phases, this pair of theories, $(X, V)$ and $(\tilde{X}, \tilde{V})$, preserve the net number of moduli and the complete charged and singlet particle spectra.

In an Abelian GLSM, there exist multiple $U(1)$ gauge fields $A^{(\alpha)}$ with $\alpha=1, \ldots, r$, two sets of chiral superfields as $\left\{X_{i} \mid i=1, \ldots, d\right\}$ with $U(1)$ charges $Q_{i}^{(\alpha)}$, and $\left\{P_{l} \mid l=\right.$ $1, \ldots, \gamma\}$ with $U(1)$ charges $-M_{l}^{(\alpha)}$. Furthermore, there are two sets of Fermi superfields: $\left\{\Lambda^{a} \mid a=1, \ldots, \delta\right\}$ with charges $N_{a}^{(\alpha)}$, and $\left\{\Gamma^{j} \mid j=1, \ldots, c\right\}$ with charges $-S_{j}^{(\alpha)}$. These charges are given in order to realize the Calabi-Yau manifolds as complete intersection hypersurfaces in ambient space (CICY) and stable, holomorphic vector bundles over them in some geometric phase. As a result, we will require the charges $Q_{i}^{(\alpha)} \geq 0$, and for each $i$, there exists at least one $r$ such that $Q_{i}^{(\alpha)}>0$. A similar assumption of (semi)positivity will also hold for the charges $S_{j}^{(\alpha)}$ and $M_{l}^{(\alpha)}$. However, in some cases, we will consider solutions in which charges $N_{a}^{(\alpha)}$ may be negative. Then the field content and charges of GLSM can be summarized in the following "charge matrix": 


\begin{tabular}{|cccc||cccc|}
\hline \multicolumn{4}{|c||}{$x_{i}$} & & & \multicolumn{3}{c|}{$\Gamma^{j}$} & & \\
\hline & & & & & & & \\
$Q_{1}^{(1)}$ & $Q_{2}^{(1)}$ & $\ldots$ & $Q_{d}^{(1)}$ & $-S_{1}^{(1)}$ & $-S_{2}^{(1)}$ & $\ldots$ & $S_{c}^{(1)}$ \\
$Q_{1}^{(2)}$ & $Q_{2}^{(2)}$ & $\ldots$ & $Q_{d}^{(2)}$ & $-S_{1}^{(2)}$ & $-S_{2}^{(2)}$ & $\ldots$ & $S_{c}^{(2)}$ \\
$\vdots$ & $\vdots$ & $\ddots$ & $\vdots$ & $\vdots$ & $\vdots$ & $\ddots$ & $\vdots$ \\
$Q_{1}^{(r)}$ & $Q_{2}^{(r)}$ & $\ldots$ & $Q_{d}^{(r)}$ & $-S_{1}^{(r)}$ & $-S_{2}^{(r)}$ & $\ldots$ & $S_{c}^{(r)}$ \\
& & & & & & & \\
\hline
\end{tabular}

\begin{tabular}{|cccc||cccc|}
\hline \multicolumn{5}{c||}{$\Lambda^{a}$} & & \multicolumn{4}{c}{$p_{l}$} \\
\hline \\
$N_{1}^{(1)}$ & $N_{2}^{(1)}$ & $\ldots$ & $N_{\delta}^{(1)}$ & $-M_{1}^{(1)}$ & $-M_{2}^{(1)}$ & $\ldots$ & $-M_{\gamma}^{(1)}$ \\
$N_{1}^{(2)}$ & $N_{2}^{(2)}$ & $\ldots$ & $N_{\delta}^{(2)}$ & $-M_{1}^{(2)}$ & $-M_{2}^{(2)}$ & $\ldots$ & $-M_{\gamma}^{(2)}$ \\
$\vdots$ & $\vdots$ & $\ddots$ & $\vdots$ & $\vdots$ & $\vdots$ & $\ddots$ & $\vdots$ \\
$N_{1}^{(r)}$ & $N_{2}^{(r)}$ & $\ldots$ & $N_{\delta}^{(r)}$ & $-M_{1}^{(r)}$ & $-M_{2}^{(r)}$ & $\ldots$ & $-M_{\gamma}^{(r)}$ \\
& & & & & & & \\
\hline
\end{tabular}

We can denote such a starting point in the geometric phase as

$$
V_{N_{1}, \ldots, N_{\delta}}\left[M_{1}, \ldots, M_{\gamma}\right] \rightarrow \mathbb{P}_{Q_{1}, \ldots Q_{d}}\left[S_{1}, \ldots, S_{c}\right] .
$$

Here the anomaly cancellation condition requires the following linear and quadratic constraints for all $\alpha, \beta=1, \ldots, r$ :

$$
\begin{aligned}
& \sum_{a=1}^{\delta} N_{a}^{(\alpha)}=\sum_{l=1}^{\gamma} M_{l}^{(\alpha)}, \quad \sum_{i=1}^{d} Q_{i}^{(\alpha)}=\sum_{j=1}^{c} S_{j}^{(\alpha)}, \\
& \sum_{l=1}^{\gamma} M_{l}^{(\alpha)} M_{l}^{(\beta)}-\sum_{a=1}^{\delta} N_{a}^{(\alpha)} N_{a}^{(\beta)}=\sum_{j=1}^{c} S_{j}^{(\alpha)} S_{j}^{(\beta)}-\sum_{i=1}^{d} Q_{i}^{(\alpha)} Q_{i}^{(\beta)} .
\end{aligned}
$$

GLSM is further described by a superpotential and a scalar potential, while the scalar potential has contributions from the F-term and D-term,

$$
\begin{aligned}
S & =\int d^{2} z d \theta\left[\sum_{j} \Gamma^{j} G_{j}\left(x_{i}\right)+\sum_{l, a} P_{l} \Lambda^{a} F_{a}^{l}\left(x_{i}\right)\right], \\
V_{F} & =\sum_{j}\left|G_{j}\left(x_{i}\right)\right|^{2}+\sum_{a}\left|\sum_{l} p_{l} F_{a}^{l}\left(x_{i}\right)\right|^{2}, \\
V_{D} & =\sum_{\alpha=1}^{r}\left(\sum_{i=1}^{d} Q_{i}^{(\alpha)}\left|x_{i}\right|^{2}-\sum_{l=1}^{\gamma} M_{l}^{(\alpha)}\left|p_{l}\right|^{2}-\xi^{(\alpha)}\right)^{2},
\end{aligned}
$$

\begin{tabular}{|c|c|c|}
\hline$G^{j}$ & $M_{2}-N_{1} \quad M_{2}-N_{2}$ & $\ldots \quad M_{2}-N_{\delta}$ \\
\hline $\begin{array}{llll}S_{1} & S_{2} & \ldots & S_{c} \\
\end{array}$ & 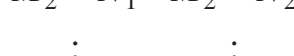 & \\
\hline & $\begin{array}{cc}: & : \\
M_{\gamma}-N_{1} & M_{\gamma}-N_{2}\end{array}$ & $M_{\gamma}-N_{\delta}$ \\
\hline
\end{tabular}

where the functions $G_{j}$ and $F_{a}^{l}$ are quasihomogeneous polynomials with degrees shown in the following matrix:
Furthermore, the function $F_{a}^{l}$ will be chosen to satisfy a transversality condition such that $F_{a}^{l}(x)=0$ only when $x_{i}=0$ for $i=1, \ldots, d$.

The $\xi^{(\alpha)} \in \mathbb{R}$ in the D-term potential is the FayetIliopoulos parameter which determines the structure of the vacuum. From Witten's original paper [20], consider the simple case with only one $U(1)$ so there is only one $\xi$ : If $\xi>0$, then it is the geometric phase, described by a vector bundle over a Calabi-Yau manifold, $V_{N_{1}, \ldots, N_{\delta}}\left[M_{1}, \ldots, M_{\gamma}\right] \rightarrow$ $X \equiv \mathbb{P}_{Q_{1}, \ldots, Q_{d}}\left[S_{1}, \ldots, S_{c}\right]$, where the Calabi-Yau manifold is defined by complete intersection hypersurfaces in weighted projective space, i.e., CICY $X=\cap_{j=1}^{c} G_{j}$ with $G_{j}\left(x_{i}\right)=0$, and the vector bundle is defined by

$$
V=\frac{\operatorname{ker}\left(F_{a}^{l}\right)}{\operatorname{im}\left(E_{i}^{a}\right)},
$$

with rank $r k(V)=\left(\delta-\gamma-r_{\mathcal{V}}\right)$ through the monad on $X$ :

$$
0 \rightarrow \mathcal{O}_{X}^{\oplus r_{\nu}} \stackrel{E_{i}^{a}}{\rightarrow} \bigoplus_{a=1}^{\delta} \mathcal{O}_{X}\left(N_{a}\right) \stackrel{F_{a}^{l}}{\rightarrow} \bigoplus_{l=1}^{\gamma} \mathcal{O}_{X}\left(M_{l}\right) \rightarrow 0
$$

If $\xi<0$, then it is the Landau-Ginzburg phase described by a superpotential,

$$
\mathcal{W}\left(x_{i}, \Lambda^{a}, \Gamma^{i}\right)=\sum_{j} \Gamma^{j} G_{j}\left(x_{i}\right)+\sum_{a} \Lambda^{a} F_{a}\left(x_{i}\right),
$$

where it would be a hybrid-type nongeometric phases if there are multiple $U(1)$ 's.

Now let us move to the target space duality. The first observation is that an exchange/relabeling of $G_{j}$ 's and $F_{a}$ 's will leave the superpotential (2.8) invariant. This observation indicates that two distinct GLSMs could "share" a nongeometric phase in which the original role of $G_{j}$ and $F_{a}$ is obscured. So the full procedure of a target space dual would be starting from a geometric phase, going to a Landau-Ginzburg phase, doing a rescaling/relabeling of the fields, and going back to the geometric phase to get a new Calabi-Yau/vector bundle configuration.

If the Landau-Ginzburg phase exists, then the rescaling procedure is as follows, for a nonvanishing $p_{l}$ and all $i=1, \ldots, k$ : 


$$
\begin{gathered}
\tilde{\Lambda}^{a_{i}}:=\frac{\Gamma^{j_{i}}}{\left\langle p_{l}\right\rangle}, \quad \tilde{\Gamma}^{j_{i}}=\left\langle p_{l}\right\rangle \Lambda^{a_{i}}, \\
\left\|\tilde{\Lambda}^{a_{i}}\right\|=\left\|\Gamma^{j_{i}}\right\|-\left\|P_{l}\right\|, \quad\left\|\tilde{\Gamma}^{j_{i}}\right\|=\left\|\Lambda^{a_{i}}\right\|+\left\|P_{l}\right\|,
\end{gathered}
$$

with $\sum_{i}\left\|G_{j_{i}}\right\|=\sum_{i}\left\|F_{a_{i}}{ }^{l}\right\|$ for anomaly cancellation. One thing to notice is that exchanging only one $F$ with one $G$ does nothing. So in all examples, two or more $F_{a}$ 's are exchanged with two or more $G_{j}$ 's. It is clear that the "relabeling" of fields at the shared Landau-Ginzburg point can mix the degrees of freedom (d.o.f.) in $h^{2,1}(X)$ and $h^{1}\left(X, \operatorname{End}_{0}(V)\right)$ in the target space dual. In the landscape [3], the dual sides match in the number of charged matter, and the total number of massless gauge singlets, where the individual number of complex, Kähler, and bundle moduli are interchanged as

$$
\begin{aligned}
& h^{*}\left(X, \wedge^{k} V\right)=h^{*}\left(\tilde{X}, \wedge^{k} \tilde{V}\right), \quad k=1,2, \ldots, r k(V), \\
& h^{2,1}(X)+h^{1,1}(X)+h_{X}^{1}\left(\operatorname{End}_{0}(V)\right) \\
& \quad=h^{2,1}(\tilde{X})+h^{1,1}(\tilde{X})+h_{X}^{1}\left(\operatorname{End}_{0}(\tilde{V})\right) .
\end{aligned}
$$

Furthermore, more general target space duality are possible such that it can also change the dimension of $h^{1,1}(\tilde{X})$. For example, if there is only one column in $G$, which is not enough to make the exchange, then a blowup of $\mathbb{P}^{1}$ on the manifold will help. This procedure leads to a dual model with an additional $U(1)$ action. In this case, it is necessary to rewrite the initial GLSM in an equivalent/ redundant way. It is always possible to introduce into the GLSM a new coordinate (i.e., a new Fermi superfield) $y_{1}$ with multidegree $\mathcal{B}$ and a new hypersurface (i.e., a chiral superfield with an opposite charge of the new Fermi superfield) $G^{\mathcal{B}}$ corresponding to a homogeneous polynomial of multidegree $\mathcal{B}$. Similar to Eq. (2.2), the above addition can be written

$V_{N_{1}, \ldots, N_{\delta}}\left[M_{1}, \ldots, M_{\gamma}\right] \rightarrow \mathbb{P}_{Q_{1}, \ldots, Q_{d}, \mathcal{B}}\left[S_{1}, \ldots, S_{c}, \mathcal{B}\right]$,

and the matrix form of such an intermediate step should be

\begin{tabular}{|ccccc||cccc|}
\hline$x_{1}$ & $\ldots$ & $x_{d}$ & $y_{1}$ & $y_{2}$ & $\Gamma^{1}$ & $\ldots$ & $\Gamma^{c}$ & $\Gamma^{B}$ \\
\hline 0 & $\ldots$ & 0 & 1 & 1 & 0 & $\ldots$ & 0 & -1 \\
$Q_{1}$ & $\ldots$ & $Q_{d}$ & $\mathcal{B}$ & 0 & $-S_{1}$ & $\ldots$ & $-S_{c}$ & $-\mathcal{B}$ \\
\hline
\end{tabular}

\begin{tabular}{|cccc||rccc|}
\hline$\Lambda^{1}$ & $\Lambda^{1}$ & $\ldots$ & $\Lambda^{\delta}$ & $p_{1}$ & $p_{2}$ & $\ldots$ & $p_{\gamma}$ \\
\hline 0 & 0 & $\ldots$ & 0 & -1 & 0 & $\ldots$ & 0 \\
$N_{1}$ & $N_{2}$ & $\ldots$ & $N_{\delta}$ & $-M_{1}$ & $-M_{2}$ & $\ldots$ & $-M_{\gamma}$ \\
\hline
\end{tabular}

Suppose that in an example there are two chosen map elements $F_{1}^{1}$ and $F_{2}^{1}$ that have been chosen to be interchanged with a defining relation $S_{1}$. In this case, we can choose the redundant new coordinate, $y_{1}$, to have charge

$$
\|\mathcal{B}\|=\left\|F_{1}^{1}\right\|+\left\|F_{2}^{1}\right\|-S_{1} .
$$

For the initial configuration, $\left\|G_{1}\right\|+\left\|G_{2}\right\|=\left\|F_{1}^{1}\right\|+\left\|F_{2}^{1}\right\|$, where $G_{1}, G_{2}$ are $S_{1}, \mathcal{B}$. Under the relabelings required in Eq. (2.9), one can choose

$$
\begin{aligned}
& \tilde{N}_{1}=M_{1}-S_{1}, \quad \tilde{N}_{2}=M_{1}-\mathcal{B}, \\
& \tilde{S}_{1}=\left\|F_{1}^{1}\right\|, \quad \tilde{\mathcal{B}}=\left\|F_{2}^{1}\right\| .
\end{aligned}
$$

Applying the field redefinitions in Eq. (2.15), we arrive at last at the new configuration,

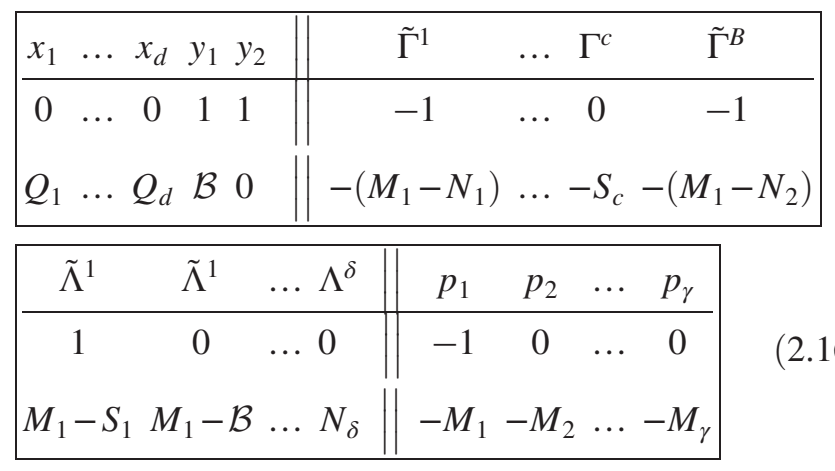

In subsequent sections, we will primarily consider examples of the latter kind in which all three types of singlet moduli-Kähler, complex structure, and bundle moduli-are interchanged in the target space duality procedure. We next turn to such an example in which both $X$ and $\tilde{X}$ are elliptically fibered.

\section{A TARGET SPACE DUAL PAIR WITH ELLIPTICALLY FIBERED CALABI-YAU THREEFOLDS}

Before we can begin investigating the consequences of $(0,2)$ target space duality for F-theory, a nontrivial first step is to establish whether examples exist in which both halves of a TSD pair in turn lead to F-theory dual geometries. In this section, we explicitly provide a first example of such a pair.

In the following example, we will find that the CY manifolds, $X$ and $\tilde{X}$, consist of two CICY threefolds $[18,21]$, each of which is fibered over (a different) complex surface $B_{2}$. These two CICY threefolds are related by a conifold transition [21] and can be constructed via the target space duality algorithm in which an additional $U(1)$ symmetry is added to the dual GLSM as in Sec. II. 


\section{A. A tangent bundle deformation}

To investigate these results, a simple starting point is given below- a dual pair for which the Calabi-Yau manifolds, $X$ and $\tilde{X}$, are related by a conifold transition. Consider the following CICY threefold, described by a so-called configuration matrix [21]:

$$
X=\left[\begin{array}{l|ll}
\mathbb{P}^{1} & 1 & 1 \\
\mathbb{P}^{2} & 1 & 2 \\
\mathbb{P}^{2} & 1 & 2
\end{array}\right]
$$

Here the columns indicate the ambient space (a product of complex projective spaces) and the degrees of the defining equations in that space. The Hodge numbers are $h^{1,1}(X)=3$ and $h^{2,1}(X)=60$. Over this manifold, we choose a simple vector bundle built as a deformation of the holomorphic tangent bundle to $X$. In this case, we will choose this bundle to be a rank 6 smoothing deformation of the reducible bundle,

$$
V_{\text {red }}=\mathcal{O}^{\oplus 3} \oplus T X
$$

The smooth, indecomposable bundle will be defined ${ }^{1}$ as a kernel $V \equiv \operatorname{ker}\left(F_{a}^{l}\right)$ via the short exact sequence

$$
0 \rightarrow V \rightarrow \bigoplus_{a=1}^{\delta} \mathcal{O}_{\mathcal{M}}\left(N_{a}\right) \stackrel{F_{a}^{l}}{\rightarrow} \bigoplus_{l=1}^{\gamma} \mathcal{O}_{\mathcal{M}}\left(M_{l}\right) \rightarrow 0,
$$

which is the simple case of Eq. (2.7) when $E_{i}^{a}=0$.

In the language of GLSM charge matrices, the manifold and rank 6 monad bundle $(X, V)$ are given by the following charge matrix:

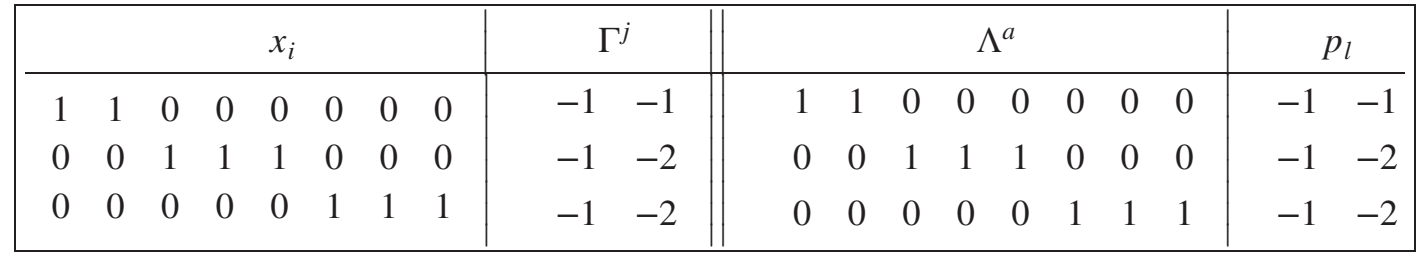

The reason that this rank 6 bundle makes for a particularly simple choice of gauge bundle is that in this case the GLSM charges associated with the manifold and the bundle are identical (as can be seen above). As a result, anomaly cancellation conditions such as the requirement that

$$
c_{2}(T X)=c_{2}(V)
$$

[realized as Eq. (2.3) in the GLSM] are automatically satisfied.

Expanding the second Chern class of the manifold in a basis of $\{1,1\}$ forms $J_{r}, r=1, \ldots, h^{1,1}$, we have

$$
c_{2}(T X)=3 J_{1} J_{2}+J_{2}^{2}+3 J_{1} J_{3}+5 J_{2} J_{3}+J_{3}^{2} .
$$

Following the standard $(0,2)$ target space duality procedure, it is easy to produce the TSD geometry $(\tilde{X}, \tilde{V})$. In this case, the duals we consider mix all three types of heterotic geometry moduli and induce an additional $U(1)$ gauge symmetry in the GLSM. As an intermediate step, we form the equivalent GLSM charge matrix with an additional $U(1)$ outlined in Sec. II (choosing $\mathcal{B}=0$ ) and introduce a repeated entry in the monad bundle

\begin{tabular}{|c|c|c|c|c|c|c|c|c|c|c|c|c|c|c|c|c|c|c|c|c|c|c|c|c|}
\hline \multicolumn{10}{|c|}{$x_{i}$} & \multicolumn{3}{|c|}{$\Gamma^{j}$} & \multicolumn{9}{|c|}{$\Lambda^{a}$} & \multicolumn{3}{|c|}{$p_{l}$} \\
\hline 1 & 1 & 0 & 0 & 0 & 0 & 0 & 0 & 0 & 0 & 0 & -1 & -1 & 1 & 1 & 0 & 0 & $0 \quad$ & 0 & $0 \quad$ & 0 & 1 & -1 & -1 & $\overline{-1}$ \\
\hline 0 & 0 & 1 & 1 & 1 & 0 & 0 & 0 & 0 & 0 & 0 & -1 & -2 & 0 & 0 & 1 & 1 & 1 & 0 & 0 & 0 & 2 & -1 & -2 & -2 \\
\hline 0 & 0 & 0 & 0 & 0 & 1 & 1 & 1 & 0 & 0 & 0 & -1 & -2 & 0 & 0 & 0 & 0 & 0 & 1 & 1 & 1 & 1 & -1 & -2 & -1 \\
\hline 0 & 0 & 0 & 0 & 0 & 0 & 0 & 0 & 1 & 1 & -1 & 0 & 0 & 0 & 0 & 0 & 0 & 0 & 0 & 0 & 0 & 0 & 0 & -1 & 0 \\
\hline
\end{tabular}
charges which does not change either the geometry of GLSM field theory. ${ }^{2}$ This leads us to the following charge matrix with a new $\mathbb{P}^{1}$ row and a new column $\Gamma^{\mathcal{B}}$ as in Eq. (2.13):

\footnotetext{
${ }^{1}$ See [22] for discussions of this deformation problem and local moduli space.

${ }^{2}$ See [3] for details of this argument.
} 
Finally, we can perform the field redefinitions in this intermediate geometry to obtain the final TSD. Here we choose two map elements - in this case, $F_{8}^{2}$ and $F_{9}^{2}$ - to be interchanged with a defining relation $G_{2}$ with degree $\left\|S_{2}\right\|=\{1,2,2\}$. Such a choice satisfies the linear anomaly cancellation (2.14) since $\left\|S_{2}\right\|+\mathbf{0}=\left\|F_{8}^{2}\right\|+\left\|F_{9}^{2}\right\|$. In the intermediate configuration, applying the field redefinitions (2.15) gives

$$
\begin{gathered}
\tilde{N}_{8}=M_{2}-S_{2}=0, \quad \tilde{N}_{9}=M_{2}, \\
\tilde{S}_{2}:=F_{9}^{2}, \quad \tilde{\mathcal{B}}:=F_{8}^{2} .
\end{gathered}
$$

This leads us at last to the dual charge matrix associated

\begin{tabular}{|c|c|c|c|c|c|c|c|c|c|c|c|c|c|c|c|c|c|c|c|c|c|c|c|c|}
\hline \multicolumn{10}{|c|}{$x_{i}$} & \multicolumn{3}{|c|}{$\Gamma^{j}$} & \multicolumn{9}{|c|}{$\Lambda^{a}$} & \multicolumn{3}{|c|}{$p_{l}$} \\
\hline 1 & & 0 & 0 & 0 & 0 & 0 & 0 & 0 & 0 & 0 & -1 & -1 & 1 & 1 & 0 & 0 & 0 & 0 & 0 & 0 & 1 & -1 & -1 & -1 \\
\hline 0 & 0 & 1 & 1 & 1 & 0 & 0 & 0 & 0 & 0 & 0 & -1 & -2 & 0 & 0 & 1 & 1 & 1 & 0 & 0 & 0 & 2 & -1 & -2 & -2 \\
\hline 0 & 0 & 0 & 0 & 0 & 1 & 1 & 1 & 0 & 0 & -1 & -1 & -1 & 0 & 0 & 0 & 0 & 0 & 1 & 1 & 0 & 2 & -1 & -2 & -1 \\
\hline 0 & 0 & 0 & 0 & 0 & 0 & 0 & 0 & 1 & 1 & -1 & 0 & -1 & 0 & 0 & 0 & 0 & 0 & 0 & 0 & 1 & 0 & 0 & -1 & 0 \\
\hline
\end{tabular}
with $(\tilde{X}, \tilde{V})$ with $h^{1,1}(\tilde{X})=4$ and $h^{2,1}(\tilde{X})=60$ :
Again, in the new configurations, the anomaly cancellation condition is satisfied (as was proved, in general, to happen in [3]). To make sure they are true target space duals, we will show that these two different geometric phases preserve the net multiplicities of charged matter, and the total number of massless gauge singlets, while the individual number of complex, Kähler, and bundle moduli is changed. First, it is clear that the low-energy gauge group $G$ in the four-dimensional gauge theory is given by the commutant of the structure group, $H$ in $E_{8} \times E_{8}$ of the bundles defined over the CY manifold. Here there is only one bundle [saturating the anomaly cancellation condition on $c_{2}(V)$ ]. We choose to embed this structure group in one of the two $E_{8}$ factors and when considering the other $E_{8}$ factor as an unbroken, hidden sector gauge symmetry.

In order to find the matter field representations, the adjoint $\mathbf{2 4 8}$ of $E_{8}$ must be decomposed under the subgroup $G \times H$. In this case, the rank 6 bundles with $c_{1}=0$ indicates that the structure group $H=S U(6)$, which leads to the charged matter spectrum, can be determined by the decomposition of $E_{8}$ into representations of the maximal subgroup $S U(2) \times S U(3) \times S U(6)$ :

$$
\begin{aligned}
\mathbf{2 4 8}_{E_{8}} \rightarrow & {[(\mathbf{3}, \mathbf{1}, \mathbf{1}) \oplus(\mathbf{1}, \mathbf{8}, \mathbf{1}) \oplus(\mathbf{1}, \mathbf{1}, \mathbf{3 5}) \oplus(\mathbf{1}, \mathbf{3}, \overline{\mathbf{1 5}})} \\
& \oplus(\mathbf{1}, \overline{\mathbf{3}}, \mathbf{1 5}) \oplus(\mathbf{2}, \mathbf{3}, \mathbf{6}) \oplus(\overline{\mathbf{2}}, \overline{\mathbf{3}}, \overline{\mathbf{6}}) \oplus(\mathbf{2}, \mathbf{1}, \mathbf{2 0})] .
\end{aligned}
$$

As a result, the multiplicity of fields in the four-dimensional theory transforming in representations of $S U(2) \times S U(3)$ is counted by those transforming in an $S U(6)$ representation over the CY. The latter are counted by the dimension of bundle valued cohomology groups, $H^{*}\left(X, \wedge^{k} V\right)$, for assorted values of $k$ (see [23,24] for details).

It is helpful to note that, for a vector bundle $V$ on a Calabi-Yau threefold $X$, the cohomology groups of the bundle and its dual are related by Serre duality as $H^{i}(X, V)=H^{3-i}\left(X, V^{*}\right)^{*}$ and when $H=S U(n)$, $H^{*}\left(X, \wedge^{k} V\right) \simeq H^{*}\left(X, \wedge^{n-k} V^{*}\right)$. Finally, a necessary condition for $\mu$ stability of the vector bundle $V$ is $h^{0}(X, V)=0$, which is satisfied for tangent bundle deformations considered here (by direct computation).

With these observations in hand, the multiplicity of the charged chiral matter spectrum of these dual pair theories can be determined by computing corresponding vector bundle valued cohomology classes on the Calabi-Yau threefold:

$$
\begin{aligned}
& (\mathbf{2}, \mathbf{3})^{\prime} \mathrm{s}: h^{1}(V)=57, \quad h^{1}(\tilde{V})=57, \\
& (\overline{\mathbf{2}}, \overline{\mathbf{3}}) \text { 's: } h^{1}\left(V^{*}\right)=0, \quad h^{1}\left(\tilde{V}^{*}\right)=0, \\
& (\mathbf{1}, \mathbf{3})^{\prime} \mathrm{s}: h^{1}\left(\wedge^{2} V\right)=115, \quad h^{1}\left(\wedge^{2} \tilde{V}\right)=115 \text {, } \\
& (\mathbf{1}, \overline{\mathbf{3}})^{\prime} \text { s: } h^{1}\left(\wedge^{2} V^{*}\right)=1, \quad h^{1}\left(\wedge^{2} \tilde{V}^{*}\right)=1 \text {, } \\
& (\mathbf{2 , 1}) \text { 's: } h^{1}\left(\wedge^{3} V\right)=2, \quad h^{1}\left(\wedge^{3} \tilde{V}\right)=2 \text {. }
\end{aligned}
$$

Furthermore, the low-energy theory has massless gauge singlets, $(1,1)$, which are counted by $h^{1}\left(V \otimes V^{*}\right)=$ $h^{1}\left(\operatorname{End}_{0}(V)\right)$. There are additional singlets, beyond those related to the complex structure and Kähler deformations of the Calabi-Yau threefold, which are counted by $h^{2,1}(X)$ and $h^{1,1}(X)$. The total number of singlet moduli are counted by

$$
\begin{aligned}
& h^{1,1}(X)+h^{2,1}(X)+h^{1}\left(\operatorname{End}_{0}(V)\right)=3+60+292=355, \\
& h^{1,1}(\tilde{X})+h^{2,1}(\tilde{X})+h^{1}\left(\operatorname{End}_{0}(\tilde{V})\right)=4+53+298=355 .
\end{aligned}
$$

From the point of view of the massless heterotic spectrum, it is clear that in the theories associated with the TSD geometries, $(X, V)$ and $(\tilde{X}, \tilde{V})$, all of the d.o.f. appear to match. 
Moreover, we have chosen this pair of geometries to have a further special property. Each CY threefold appearing in the dual pair exhibits an elliptic fibration structure. As a result, by the arguments laid out in Sec. II, we expect each heterotic background in the pair to lead to its own F-theory dual.

A closer inspection yields the following elliptic fibration structures:

$$
\pi_{h}: X \stackrel{\mathbb{E}}{\rightarrow} \mathbb{P}^{2} \quad \text { and } \quad \tilde{\pi}_{h}: \tilde{X} \stackrel{\mathbb{E}}{\rightarrow} d P_{1} .
$$

The fibrations of $X$ and $\tilde{X}$ can be seen very explicitly in the form of the complete intersection descriptions of the manifolds (so-called obvious fibrations [25]). Below we use dotted lines to separate the "base" and "fiber" of the manifold:

$$
X=\left[\begin{array}{l|ll}
\mathbb{P}^{1} & 1 & 1 \\
\mathbb{P}^{2} & 1 & 2 \\
\mathbb{P}^{2} & 1 & 2
\end{array}\right], \quad \tilde{X}=\left[\begin{array}{l|lll}
\mathbb{P}^{1} & 0 & 1 & 1 \\
\mathbb{P}^{2} & 0 & 1 & 2 \\
\mathbb{P}^{2} & 1 & 1 & 1 \\
\mathbb{P}^{1} & 1 & 0 & 1
\end{array}\right]
$$

where the base for the elliptically fibered $X$ is $B_{2}=\mathbb{P}^{2}$ (the bottom row of the configuration matrix), while the $d P_{1}$ base for $\tilde{X}$ is given as $\tilde{B_{2}}=\left[\begin{array}{l}\mathbb{P}^{2} \mid 1 \\ \mathbb{P}^{1} \mid 1\end{array}\right]$.

Employing the techniques of $[26,27]$, we find that the fibrations in both $X$ and $\tilde{X}$ in fact admit rational sections and, as a result, are elliptically fibered (as opposed to genus-1 fibered only). Moreover, each fibration contains two rational sections (i.e., a higher rank Mordell-Weil group). In an abuse of notation, we will use $\sigma_{i}$ to denote both sections of the elliptic fibrations of $X$ and $\tilde{X}$ and the associated Kähler forms dual to the divisors. In terms of the basis of the Kähler $(1,1)$-forms $J_{r}$ inherited from the ambient space factors $\mathbb{P}_{r}^{n}$ of each CICY threefold,

$$
\begin{array}{ll}
\sigma_{1}(X)=-J_{1}+J_{2}+J_{3}, & \sigma_{2}(X)=2 J_{1}-J_{2}+5 J_{3} \\
\sigma_{1}(\tilde{X})=-J_{1}+J_{2}+J_{3}, & \sigma_{2}(\tilde{X})=2 J_{1}-J_{2}+4 J_{3}+J_{4},
\end{array}
$$

with a choice of zero section for each manifold from the set above, the CY threefold can, in principle, be put into Weierstrass form $[28,29]$. For explicit techniques to carry out this process, we refer the reader to [26].

In summary then, we have produced an explicit example of a TSD pair in which both sides are elliptically fibered manifolds, admitting four-dimensional, $\mathcal{N}=1$ F-theory duals in principle. This is an important point of principle, since we have demonstrated that some F-theory correspondence should exist for the dual F-theory effective field theories (EFTs). In practice, however, it should be noted that explicitly determining the F-theory duals for the geometries given above is difficult. We will begin untangling this process explicitly in Sec. IV.

For now, we close this example by observing an interesting feature of the TSD pair above: Since we began with a deformation of the tangent bundle, the associated $(0,2)$ GLSM admits a $(2,2)$ locus. However, in the TSD geometry, the bundle we obtain is no longer manifestly a holomorphic deformation of the tangent bundle on $\tilde{X}$. It remains an open question whether this second theory admits a $(2,2)$ locus in some subtle way. For the moment, we will turn to one further TSD pair in which neither vector bundle is related to the tangent bundle.

\section{B. More general vector bundles}

Here we present a second example in which the same CY manifolds appear, but with different vector bundles. Once again, we start with the GLSM charge matrix determining the pair $(\mathrm{X}, \mathrm{V})$ as in Eq. (3.16), where in this time we have a rank 4 bundle with structure group $S U(4)$ :

\begin{tabular}{|cccccccc||cc||cccccc|cc|}
\hline \multicolumn{7}{|c|}{$x_{i}$} & \multicolumn{1}{|c|}{$\Gamma^{j}$} & \multicolumn{7}{|c|}{$\Lambda^{a}$} & \multicolumn{2}{|c}{$p_{l}$} \\
\hline 1 & 1 & 0 & 0 & 0 & 0 & 0 & 0 & -1 & -1 \\
0 & 0 & 1 & 1 & 1 & 0 & 0 & 0 & -1 & -2 & 0 & 0 & 0 & 0 & 1 & -1 & -1 \\
0 & 0 & 0 & 0 & 0 & 1 & 1 & 1 & -1 & -2 & 0 & 1 & 1 & 0 & 0 & 2 & -2 & -2 \\
0 & 0 & 0 & 1 & 1 & 1 & -2 & -1 \\
\hline
\end{tabular}

In this case, the second Chern class of $(X, V)$ is different than Eq. (3.6):

$$
c_{2}(V)=2 J_{1} J_{2}+J_{2}^{2}+2 J_{1} J_{3}+4 J_{2} J_{3}+J_{3}^{2} .
$$

However, in this case, $c_{2}(V) \leq c_{2}(T X)$, and thus it is expected that this bundle could be embedded in one factor of the $E_{8} \times E_{8}$ heterotic string, where another bundle $V^{\prime}$ is embedded in the second factor. By completing the geometry in this way, with $c_{2}(V)+c_{2}\left(V^{\prime}\right)=$ $c_{2}(T X)$, the anomaly cancellation conditions can be satisfied (alternatively, NS5-/M5- branes might be considered).

Following the standard procedure described above, the target space duality data is given by $(\tilde{X}, \tilde{V})$ with the following charge matrix: 


\begin{tabular}{|c|c|c|c|c|c|c|c|c|c|c|c|c|c|c|c|c|c|c|c|c|}
\hline \multicolumn{10}{|c|}{$x_{i}$} & \multicolumn{3}{|c|}{$\Gamma^{j}$} & \multicolumn{6}{|c|}{$\Lambda^{a}$} & \multicolumn{2}{|c|}{$p_{l}$} \\
\hline 1 & 1 & 0 & 0 & 0 & 0 & 0 & 0 & 0 & 0 & 0 & -1 & -1 & 1 & 0 & 0 & 0 & 0 & 1 & -1 & -1 \\
\hline 0 & 0 & 1 & 1 & 1 & 0 & 0 & 0 & 0 & 0 & 0 & -1 & -2 & 0 & 1 & 1 & 0 & 0 & 2 & -2 & -2 \\
\hline 0 & 0 & 0 & 0 & 0 & 1 & 1 & 1 & 0 & 0 & -1 & -1 & -1 & 0 & 0 & 0 & 1 & 0 & 2 & -2 & -1 \\
\hline 0 & 0 & 0 & 0 & 0 & 0 & 0 & 0 & 1 & 1 & -1 & 0 & -1 & 0 & 0 & 0 & 0 & 1 & 0 & -1 & 0 \\
\hline
\end{tabular}

Here the second Chern classes of the tangent bundle and the monad vector bundle are, respectively,

$$
\begin{aligned}
c_{2}(T X)= & 3 J_{1} J_{2}+J_{2}^{2}+2 J_{1} J_{3}+3 J_{2} J_{3}+J_{1} J_{4} \\
& +2 J_{2} J_{4}+2 J_{3} J_{4}, \\
c_{2}(V)= & 2 J_{1} J_{2}+J_{2}^{2}+J_{1} J_{3}+2 J_{2} J_{3}+J_{1} J_{4} \\
& +2 J_{2} J_{4}+2 J_{3} J_{4},
\end{aligned}
$$

which could also satisfy the $c_{2}$ matching condition with the addition of a hidden sector bundle.

In this background, the bundle structure group of $H=$ $S U(4)$ breaks $E_{8}$ to $S O(10)$. As above, the charged matter content can be determined by the decomposition under $S O(10) \times S U(4):$

$$
\mathbf{2 4 8}_{E_{8}} \rightarrow(\mathbf{1}, \mathbf{1 5}) \oplus(\mathbf{1 0}, \mathbf{6}) \oplus(\overline{\mathbf{1 6}}, \overline{\mathbf{4}}) \oplus(\mathbf{1 6}, 4) \oplus(45,1) .
$$

The multiplicity of the spectrum is then determined via bundle valued cohomology as

$$
\begin{aligned}
\text { 16's : } h^{1}(V) & =48, & & h^{1}(\tilde{V})=48, \\
\overline{\text { 16}} \text { 's : } h^{1}\left(V^{*}\right) & =0, & & h^{1}\left(\tilde{V}^{*}\right)=0, \\
\text { 10's : } h^{1}\left(\wedge^{2} V\right) & =0, & & h^{1}\left(\wedge^{2} \tilde{V}\right)=0 .
\end{aligned}
$$

Furthermore, the counting of the gauge singlets appearing in this TSD pair match as well:

$$
\begin{aligned}
& h^{1,1}(X)+h^{2,1}(X)+h^{1}\left(\operatorname{End}_{0}(V)\right)=3+60+159=222 \\
& h^{1,1}(\tilde{X})+h^{2,1}(\tilde{X})+h^{1}\left(\operatorname{End}_{0}(\tilde{V})\right)=4+53+165=222 .
\end{aligned}
$$

With these two examples in hand, it is clear that at least the first question outlined in Sec. I can be answered in the positive. Heterotic TSD pairs can indeed be found in which both halves of the dual pair exhibit elliptic fibrations. However, it is clear that the manifolds in our examples above are not in simple Weierstrass form (and exhibit a higher rank Mordell-Weil group); as a result, their F-theory dual geometries may be difficult to determine using standard tools. We review some of these tools in the subsequent sections before returning to the two examples above in Sec. VI.

\section{INDUCING A DUALITY IN F-THEORY}

\section{A. Essential tools for heterotic/F-theory duality}

In type IIB superstring theory, the axion-dilaton transforms under $S L(2, Z)$ while leaving the action invariant. However, it is frequently assumed that the string coupling $g_{s}$ vanishes and the backreaction from 7-branes is ignored. As a result, many important nonperturbative aspects of the string compactification which are crucial both conceptually and phenomenologically are missing. This is exactly where F-theory arises as a proper description of orientifold IIB theory with $(p, q)$ 7-branes and varying finite string coupling (i.e., axion-dilaton). The classical $S L(2, Z)$ self-dual symmetry of type IIB theory acting on the axion-dilaton is identified as the modular group of a onedimensional complex torus $T^{2}$ and as the complex structure of a fictitious elliptic curve. In this way, we formally attach an elliptic curve at each point of the type IIB spacetime and promote the ten-dimensional IIB theory to auxiliary 12dimensional F-theory. This structure defines a genus-1 or elliptic fibration. The locus where the fiber degenerates is where the 7-brane is wrapped in the internal CY. F-theory realizes a remarkable synthesis of geometry and field theory in that the structure of the 7-branes/gauge sector, the matter content, and the Yukawa couplings are all encoded in the geometry of the fibration structure, and the backreaction of these branes is taken into account.

There is no description of F-theory as a fundamental theory, but rather as duals to other theories. A concrete example would be an eight-dimensional duality [10], i.e., F-theory compactified on $K 3$ is dual to type IIB on $S^{2}$ with 24 7-branes turned on, which is also dual to the heterotic on $T^{2}$. The duality between F-theory and the heterotic is described further as F-theory compactified on $K 3$ fibered Calabi-Yau $(n+1)$-fold is dual to the $E_{8} \times E_{8}$ heterotic string compactified on the Calabi-Yau $n$-fold which is elliptically fibered on the same $(n-1)$-fold base:

(i) Heterotic: $\pi_{h}: X_{n} \stackrel{\mathbb{E}}{\rightarrow} B_{n-1}$ elliptic fibration,

(ii) F-theory: $\pi_{f}: Y_{n+1} \stackrel{K 3}{\rightarrow} B_{n-1}$, where $\rho_{f}: Y_{n+1} \stackrel{\mathbb{E}}{\rightarrow} B_{n}$, $\sigma_{f}: B_{n} \stackrel{\mathbb{P}^{1}}{\rightarrow} B_{n-1}$.

The paired heterotic/F-theory geometries given above involves both elliptic and $K 3$ fibered manifolds. In 
particular, the F-theory geometry $Y_{n+1}$ must be compatibly $K 3$ and elliptically fibered. The requirement of these two fibrations implies that $Y_{n+1}$ should also be elliptically fibered over a complex $n$-dimensional base $B_{n}$ which is in turn rationally fibered. The existence of a section in any two of the fibration structures is enough to guarantee the existence of a section in the third fibration (i.e., if $\rho_{f}$ and $\sigma_{f}$ both admit sections, then so does the fibration $\pi_{f}$ ).

With different numbers of $n$ 's, there are theories in different dimensions. Specifically, $n=1,2,3$ will lead to $8 \mathrm{D}, 6 \mathrm{D}$, and $4 \mathrm{D}$ respectively. When $n=1$, the $(n-1)$-fold base $B_{n-1}$ is a point; when $n=2$, it is a $\mathbb{P}^{1}$. In the $4 \mathrm{D}$ case, the duality can be written as

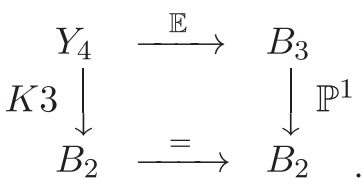

By the fibration structure of the CY fourfold (4.1), the base $B_{3}$ must be $\mathbb{P}^{1}$ fibered. As in [12], such a $\mathbb{P}^{1}$ bundle can be defined as the projectivization of two line bundles,

$$
B_{3}=\mathbb{P}(\mathcal{O} \oplus \mathcal{L}),
$$

where $\mathcal{O}$ is the trivial bundle and $\mathcal{L}$ is a general line bundle on $B_{2}$. In this case, the topology of $B_{3}$ is completely fixed by the choice of line bundle $\mathcal{L}$, and we can define a $(1,1)$ form on $B_{2}$ as $T=c_{1}(\mathcal{L})$. A special case would be $R=c_{1}(\mathcal{O}(1))$, where $\mathcal{O}(1)$ is a bundle that restricts to the usual $\mathcal{O}(1)$ on each $\mathbb{P}^{1}$ fiber. They satisfy the relation $R(R+T)=0$ in cohomology class, which indicates that the two corresponding sections do not intersect with each other. This kind of twist allows us to matching the d.o.f. in the four-dimensional heterotic/F-theory dual pairs.

On the $E_{8} \times E_{8}$ heterotic side, the vector bundle can be decomposed as $V=V_{1} \oplus V_{2}$, and the curvature splits as

$$
c_{2}(V)=\frac{1}{30} \operatorname{Tr} F_{i}^{2}=\eta_{i} \wedge \sigma+\xi_{i},
$$

where $\eta_{i}, \xi_{i}$ are pullbacks of 2-forms and 4-forms on $B_{2}, \sigma$ is the Poincare dual to the section of the elliptic fibration $\pi_{h}: X_{3} \stackrel{\mathbb{E}}{\rightarrow} B_{2}$. For any CY threefold in Weierstrass form as described above, $c_{2}\left(T X_{3}\right)=12 c_{1}\left(B_{2}\right) \wedge \omega_{\hat{0}}+\left(c_{2}\left(B_{2}\right)+\right.$ $\left.11 c_{1}\left(B_{2}\right)^{2}\right)$ [30]. The heterotic Bianchi identity requires $\eta_{1}+\eta_{2}=12 c_{1}\left(B_{2}\right)$, which enables us to parametrize a solution as

$$
\eta_{1,2}=6 c_{1}\left(B_{2}\right) \pm T^{\prime},
$$

where $T^{\prime}$ is a $(1,1)$-form on $B_{2}$. By studying the $4 \mathrm{D}$ effective theories of these dual heterotic/F-theory compactifications, it is straightforward to determine that the defining $(1,1)$-forms $T, T^{\prime}$ are identical to each other
$T=T^{\prime}$. Then the $(1,1)$-form $T$ is referred to as the so-called twist of the $\mathbb{P}^{1}$ fibration and is the crucial defining data of the simplest classes of heterotic/F-theory dual pairs. Moreover, this duality maps dependences on a particular method of constructing Mumford polystable vector bundles, the spectral cover construction.

\section{B. Spectral cover construction}

To find the dual F-theory model of a specific heterotic model, we need a description of the moduli space of stable degree zero vector bundles over elliptically fibered manifolds (the standard formulation works for Weierstrass fibration, but it can be generalized to other types of elliptic fibrations) in terms of two "pieces", which are called spectral data. This can be done by Fourier-Mukai transform, and we briefly review it in the following. (FourierMukai is an important type of functor between derived categories, but to avoid unnecessary technicalities, we restrict ourselves to the following special type defined by Poincaré bundle, and we ignore general discussions. The interested reader can refer to $[31,32] .^{3}$ )

The Fourier-Mukai transform takes the stable bundles of degree zero and rank $n$ over $X$ and gives a torsion sheaf (rank 0) degree $n$ over the (compactified) Jacobian fibration $\tilde{X} \sim X$. More precisely, considering the following fiber product and the natural projections,

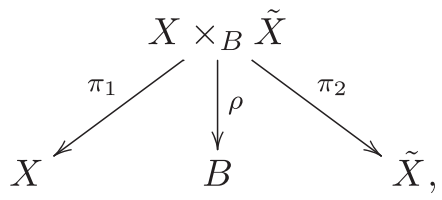

the Fourier-Mukai (FM) transform is defined as ${ }^{4}$

$$
F M^{1}(V)=\mathbf{R}^{1} \pi_{2 *}\left(\pi_{1}^{*} V \otimes \mathcal{P}^{*}\right) .
$$

We emphasize again that (compactified) Jacobians of irreducible elliptic curves are isomorphic to the elliptic curve; therefore in the Weierstrass fibration we have $X \sim \tilde{X}$. In Eq. (4.5), $\mathcal{P}$ is the Poincaré bundle,

$$
\mathcal{P}=\mathcal{O}\left(\Delta-\sigma_{1} \times \tilde{X}-X \times \sigma_{2}\right) \otimes \rho^{*} \mathcal{K}_{B},
$$

where $\Delta$ is the diagonal divisor in $X \times_{B} \tilde{X}$, and $\sigma_{1}$ and $\sigma_{2}$ are sections of the first and second factors, respectively. $R^{1} \pi_{*}$ is the first derived pushforward. Roughly speaking, the presheaf corresponding to $R \pi_{*} \mathcal{F}$ over an open set $U$ is

\footnotetext{
${ }^{3}$ Please note that we restrict ourselves to $S U(N)$ (degree zero, and stable) vector bundles over a Weierstrass elliptic fibration.

${ }^{4}$ In some sense, it is similar to the Fourier transform. In that case, one starts with a function $f(x)$ defined in a space $X$, then we pull back the function into a larger space $X \times Y$, multiply by a kernel $e^{i x \cdot y}$, and integrate over $x$ to get a function in $Y$. The pushforward action is similar to the integration over the fibers.
} 
isomorphic to $H^{1}\left(\pi^{-1} U, \mathcal{F}\right)$ (look at Sec. III. 1 of [33] and [34]). Note that the restriction of stable and degree zero vector bundles over generic fibers will be a semistable bundle with degree zero and the same rank [35]. Then a well-known theorem proved by Atiyah ${ }^{5}$ [36] tells us the general form of restriction of the vector bundle over a generic smooth elliptic curve $E{ }^{6}$

$$
\begin{aligned}
& \left.V\right|_{E}=\bigoplus_{i} \mathcal{E}_{i} \otimes \mathcal{O}_{E}\left(p_{i}-\sigma\right), \\
& \sum_{i} \operatorname{rank}\left(\mathcal{E}_{i}\right) \times p_{i}=0 .
\end{aligned}
$$

The second condition is imposed because of the "S" of the $S U(N)$ gauge group (note that the sum is over the group law of the elliptic curve), and $\mathcal{E}_{i}$ is constructed inductively by extending it with a trivial bundle,

$$
0 \rightarrow \mathcal{O}_{E} \rightarrow \mathcal{E}_{i} \rightarrow \mathcal{E}_{i-1} \rightarrow 0
$$

Therefore using Eq. (4.7), it is easy to show that the stalk of the Fourier-Mukai transform of $V$ over some generic point $p \in \tilde{X}$ takes the following form (the isomorphism can be proved by the final theorem in Sec. III. 12 of [33]):

$$
F M^{1}(V)_{p} \sim H^{1}\left(E_{p}, \bigoplus_{i} \mathcal{E}_{i} \otimes \mathcal{O}_{E}\left(p_{i}-p\right)\right) .
$$

It is clear that Eq. (4.9) is nonzero only at point $p$ that are coincident with one of the points $p_{i}$. To see this, consider the $\mathcal{E}_{2}$. By definition, we have

$$
0 \rightarrow \mathcal{O}_{E} \rightarrow \mathcal{E}_{2} \rightarrow \mathcal{O}_{E} \rightarrow 0
$$

If we multiply the whole sequence with $\mathcal{O}_{E}\left(p_{i}-p\right)$, the first cohomology of $\mathcal{E}_{2} \otimes \mathcal{O}\left(p_{i}-p\right)$ will be zero, since $\mathcal{O}\left(p_{i}-p\right)$ is a line bundle over the elliptic curve corresponding to a degree zero, but noneffective, divisor (so it does not have a global section, and by Riemann-Roch, has a trivial first cohomology). By induction, we can get similar conclusions for a general $\mathcal{E}_{i}$ in Eq. (4.7).

Therefore the Fourier-Mukai sheaf $F M^{1}(V)$ is supported on an $n$-sheeted cover, $\mathcal{S}$, of the base (possibly a nonreduced and/or reducible scheme), which is called a spectral cover. To be more clear, consider a special case of Eq. (4.7),

$$
\left.V\right|_{E}=\bigoplus_{i=1}^{n} \mathcal{O}\left(p_{i}-\sigma\right)
$$

\footnotetext{
${ }^{5}$ Intuitively, one can argue that to construct a nontrivial flat bundle over a torus, the only way is to embed the fundamental group of the torus in the Lie group, i.e., turn on the Wilson lines [12].

${ }^{6}$ Note that $\sum_{i} \operatorname{rank}\left(\mathcal{E}_{i}\right)=\operatorname{rank}(V)$.
}

such that the points $p_{i}$ are all different. In fact, every semistable bundle over $E$ is $S$ equivalent to the direct sum written above. In this situation, $\mathcal{S}$ is a nondegenerate surface (i.e., nonreduced as a scheme), and by Eq. (4.9), the rank of the Fourier-Mukai sheaf when restricted over $\mathcal{S}$ is 1 . Therefore the Fourier-Mukai transform of the vector bundle is described by an $n$-sheeted cover of the base $\mathcal{S}$, and a sheaf (called a spectral sheaf) over that, where, if $\mathcal{S}$ is nondegenerate, the restriction of the spectral sheaf will be a line bundle $\mathcal{L}$ over $\mathcal{S}^{7}$

The next question is how the vector bundle can be reconstructed from the spectral data described above. This can be done by using the inverse "functor", which simply is

$$
V=\pi_{1 *}\left(\pi_{2}{ }^{*} F M^{1}(V) \otimes \mathcal{P}\right) .
$$

The final point is that, technically, Fourier-Mukai is the equivalence of the derived category of coherent sheaves on $X$ and the derived category coherent sheaves on $\tilde{X}$ (at least when $X$ and $\tilde{X}$ are smooth). It roughly means that the Fourier-Mukai transform gives a "one-to-one" relation between the "space of stable vector bundles $V$ " and the "space of spectral data $(\mathcal{S}, \mathcal{L})$." The logic is that the latter seems easier to study than the original bundles.

In principle, it is possible to find the F-theory dual by using the spectral data. We review this briefly in the following. Suppose that we have two vector bundles $\left(V_{1}, V_{2}\right)$ over a Weierstrass elliptically fibered manifold $X$ (suppose that there are no NS5-branes). Then heterotic anomaly cancellation requires

$$
c_{2}\left(V_{1}\right)+c_{2}\left(V_{2}\right)=c_{2}(X) .
$$

Then the second Chern classes (which can be computed using the Grothendieck-Riemann-Roch theorem if we have the spectral data [12]) can generally be written as

$$
\begin{aligned}
c_{2}\left(V_{i}\right) & =\sigma \eta_{i}+\omega_{i}, \\
\eta_{i} & =6 C_{1}\left(B_{H}\right) \pm T,
\end{aligned}
$$

where $\eta_{i}$ is a divisor in the base $\left(B_{H}\right)$, and $\omega_{i}$ is the intersection of two divisors in $B_{H}$. Also by using the same method, it is not too hard to show that the divisor class of the spectral cover of $V_{i}$ is given by

$$
[\mathcal{S}]=n_{i} \sigma+\eta_{i} \text {. }
$$

Now the first statement about the heterotic and F-theory duality is that the topology of the base manifold of

\footnotetext{
${ }^{7}$ More precisely, the rank of the spectral sheaf over the modified support (a scheme defined by 0th Fitting ideal [31]) is 1 . If, for example, the support is defined as $z^{2}=0$, the rank of the spectral sheaf over the topological support, $z=0$ locus, is 2 , but over the modified support, which roughly looks like two copies of $z=0$ infinitesimally close to each other, the rank of the sheaf is 1 .
} 
the F-theory Calabi-Yau is fixed by the "twist" $T$ in Eq. (4.12) as

$$
B_{F}=\mathbb{P}\left(\mathcal{O}_{B_{H}} \oplus \mathcal{O}_{B_{H}}(T)\right) .
$$

The second statement is that the complex structure of $\mathcal{S}$ (partially) fixes the complex structure of the Calabi-Yau on the F-theory side. It is easier to describe this with an example. Suppose that we have an $S U(2)$ bundle $V$ and that its spectral cover is nondegenerate,

$$
S=a_{0} z^{2}+a_{2} x .
$$

Since one of the $E_{8}$ factors breaks down to $E_{7}$, we should have an $E_{7}$ singularity in the F-theory geometry, which is described by the following Weierstrass equation,

$$
\begin{aligned}
Y^{2} & =X^{3}+F(u, z) x+G(u, z), \\
F & =\Sigma_{i=1}^{8} F_{i}(z) u^{i}, \\
G & =\Sigma_{i=1}^{12} G_{i}(z) u^{i} .
\end{aligned}
$$

where $u$ is the affine coordinate of the $\mathbb{P}^{1}$ fiber of Eq. (4.14), and $z$ is the "collective" coordinate for $B_{H}$. Now the conjectured duality tells us that the corresponding $E_{7}$ singularity should be located near $u=0$; therefore $F_{0}=$ $F_{1}=F_{2}=0$ and $G_{0}=\cdots=G_{4}=0$. Also $a_{0}$ is identified with $G_{5}$, and $a_{2}$ with $F_{3}$.

The other vector bundle (which is embedded in the other $E_{8}$ factor) determines the singularity near $u \rightarrow \infty$, and higher polynomials $\left(F_{5}, \ldots\right.$ and $\left.G_{7}, \ldots\right)$ are determined by the spectral cover of that vector bundle (which we did not write here). The middle polynomials $F_{4}$ and $G_{6}$ are determined by the heterotic Weierstrass equation.

The last piece of data which remains is the spectral sheaf $\mathcal{L}$, which is an element of the Picard group $\operatorname{Pic}(S)$. The "space of line bundles" itself is made up of two pieces, the "discrete" part $H^{1,1}(S)$, and the "continuous" part, which is $J(S)$ [the space of degree zero (flat) line bundles],

$$
0 \rightarrow J(S) \rightarrow \operatorname{Pic}(S) \rightarrow H_{\mathbb{Z}}^{1,1}(S) \rightarrow 0 .
$$

In $6 \mathrm{D}$ theories, the discrete part can be fixed uniquely by using the Fourier-Mukai transform, and the Jacobian of the curve is mapped to the intermediate Jacobian of the CalabiYau threefold in F-theory. In type IIA or M-theory language, the "space of three forms", $H^{3}(X, \mathbb{R}) / H^{3}(X, \mathbb{Z})$, is described by the intermediate Jacobian $[12,37,38]$.

The situation in $4 \mathrm{D}$ theories is even more complicated. In such cases, it is possible to have nontrivial 4-form fluxes which can be introduced in various (equivalent) ways. One way is to define is as 4-form induced by the field strength of the 3-form in the M-theory limit. Another way is to define as a (1,1)-form flux over the 7-branes wrapping the divisors in the base times another (1,1)-form localized around the 7-brane locus $[39,40]$. In general, the 4-flux data is parametrized by the Deligne cohomology (see the lectures [41] and references therein)

$0 \rightarrow J^{2}\left(\hat{X}_{4}\right) \rightarrow H_{D}^{4}\left(\hat{X}_{4}, \mathbb{Z}(2)\right) \rightarrow H_{\mathbb{Z}}^{2,2}\left(\hat{X}_{4}\right) \rightarrow 0$,

where $\hat{X}_{4}$ is the resolved geometry in the M-theory limit, and $J^{2}$ is the intermediate Jacobian,

$$
J^{2}\left(\hat{X}_{4}\right)=H^{3}\left(\hat{X}_{4}, \mathbb{C}\right) /\left(H^{3,0}(\hat{X}) \oplus H^{2,1}\left(\hat{X}_{4}\right)\right),
$$

which corresponds to the space of flat 3-forms in M-theory. The third, and most difficult, part of the heterotic F-theory duality is that the continuous part of the spectral sheaf data $J(S)$ maps to $J^{2}\left(X_{4}\right)$, and the discrete part $H^{1,1}(S)$ [which is determined by the divisor class (the first Chern class) of the spectral line bundle] maps to the discrete part of the 4-flux data $H^{2,2}\left(\hat{X}_{4}\right)$.

\section{WARM-UP: HETEROTIC/F-THEORY DUALITY IN SIX DIMENSIONS}

In this section, we will begin in earnest the process of attempting to determine the induced duality in F-theory given by TSD and whether the multiple fibration conjecture outlined in previous sections could be a viable realization. In this simpler context, both the geometry of the F-theory compactification and the process of reparametrizing (i.e., performing a FM transform) of the heterotic data are more readily accomplished.

To begin, it should be noted that, in the context of heterotic target space duals, we will consider smooth geometries (i.e., smooth bundles over $K 3$ manifolds) in the large volume, perturbative limit of the theory. We will consider solutions without NS5-branes so that the sixdimensional theory exhibits a single tensor multiple (associated with the heterotic dilaton) (see [42] for a review). Within the context of six-dimensional F-theory EFTs with a single tensor and a heterotic dual, it is clear that we must restrict ourselves to CY threefolds that are elliptically fibered over Hirzebruch surfaces:

$$
\pi_{f}: Y_{3} \rightarrow \mathbb{F}_{n}
$$

with $n \leq 12[9,43]$.

It is our goal in this section to test the multiple fibration conjecture in the context of target space duality. At the level of GLSMs, TSD in heterotic compactifications on $K 3$ works mechanically exactly as in the case of CY threefolds. However, the associated geometry is dramatically simpler. It is clear that the two TSD GLSMs will parametrize at best two different descriptions of a $K 3$ surface, and that the process must by construction preserve the second Chern class of the vector bundle $V$ over $K 3$ (see [3] for a proof valid either for CY two- or threefolds). Since the massless spectrum of the six-dimensional heterotic theory compactified on a smooth $K 3$ is entirely determined by the rank 
and the second Chern class of $V$ (see, e.g., [11]), ${ }^{8}$ it is clear that TSD is only a simple rewriting of the same geometry and six-dimensional EFT.

However, there remains something interesting to compare to in that it can still be asked: Does the concrete process of heterotic TSD duality in six dimensions correspond to exchanging $K 3$ fibrations in the dual F-theory geometry? In the context of F-theory threefolds that are elliptically fibered over a Hirzebruch surface as described above, there is in fact only one geometry where multiple $K 3$ fibrations can arise. It was established in the very first papers on F-theory $[9,43]$ that, in order to have different $K 3$ fibrations within a CY threefold with a perturbative heterotic dual, the base twofold must be $F_{0}$. Indeed, the remarkable observation of Morrison and Vafa was that the existence of two $K 3$ fibrations in $\pi_{f}: Y_{3} \rightarrow \mathbb{F}_{0}$ (only a simple relabeling in F-theory) was dual to a highly nonperturbative heterotic/heterotic duality discovered by Duff et al. [44].

With these observations in mind, we can immediately make several observations. To begin, we must recall that a base manifold for the F-theory fibration of $\mathbb{F}_{n}$ in this context is correlated to bundles with $c_{2}=12 \pm n$ in the $E_{8} \times E_{8}$ heterotic dual. Thus, we have as follows.

(a) For any purely perturbative heterotic TSD pair in six dimensions with $c_{2}(V)=c_{2}(\tilde{V}) \neq 12$, $(0,2)$ TSD cannot correspond to multiple fibrations in $F$-theory (since as described above, such multiple $K 3$ fibrations arise only for $n=0$ ).

(b) With the point above, we have established that, in general, the multiple fibration conjecture outlined in the Introduction must be false in general, at least in the six-dimensional heterotic theories.

(c) This demonstrates that not all TSD pairs can be described by F-theory multiple fibrations, but the converse question-namely, can multiple fibrations in F-theory give rise to dual heterotic TSD pairs?-in principle remains open.

Thus, in this section we chose to look at the last point in closer detail by considering an example of a TSD pair over $K 3$ in which $c_{2}(V)=c_{2}(\tilde{V})=12$ (the so-called symmetric embedding), corresponding to an $\mathbb{F}_{n}=\mathbb{P}^{1} \times \mathbb{P}^{1}$ base in Ftheory. This will make it possible, at least in principle, for us to consider the two duals.

\section{A. Spectral cover of monads}

To begin, we observe that since the vector bundles defined by GLSMs (in their geometric phases) are usually presented as monads [19], we must deal with how to convert this description of a bundle into one compatible with heterotic/F-theory duality. As discussed in Sec. IV, it is necessary to perform an FM transform to compute the

\footnotetext{
${ }^{8}$ And the moduli space of stable sheaves over $K 3$ with fixed Chern character has only one component.
}

spectral cover in this case. As a result, here we briefly review the method first introduced in [45].

Suppose we are given a general monad such as

$$
0 \rightarrow \mathcal{V} \rightarrow \mathcal{H} \stackrel{F}{\rightarrow} \mathcal{N} \rightarrow 0
$$

where $\mathcal{H}$ and $\mathcal{F}$ are direct sums of line bundles of appropriate degree. If we assume that $\mathcal{V}$ is stable and of degree zero, then from the previous subsection we know that its restriction over a generic elliptic fiber will look like $\oplus_{i} \mathcal{O}\left(p_{i}-\sigma\right)$. So if we twist the whole monad by $\mathcal{O}(\sigma)$,

$$
\tilde{\mathcal{V}}:=\left.\mathcal{V} \otimes \mathcal{O}(\sigma)\right|_{E}=\bigoplus_{i} \mathcal{O}\left(p_{i}\right)
$$

Each factor has only one global section over the fiber, and it becomes zero exactly at the point $p_{i}$, which is the intersection of the spectral cover with the fiber. So the idea is to try to find the global section of the twisted vector bundle over elliptic fibers, then check at what points the dimension of the vector space generated by the global sections drops. To illustrate how this can be done explicitly, first consider twisting the full monad sequence by $\mathcal{O}(\sigma)$,

$$
0 \rightarrow \tilde{\mathcal{V}} \rightarrow \tilde{\mathcal{H}} \stackrel{F}{\rightarrow} \tilde{\mathcal{N}} \rightarrow 0
$$

and follow this by next taking the action of the left exact functor $\pi_{*}$ on the above sequence ( $\bar{F}$ is the induced map, corresponding to $F$ ):

$$
0 \rightarrow \pi_{*} \tilde{\mathcal{V}} \rightarrow \pi_{*} \tilde{\mathcal{H}} \stackrel{\bar{F}}{\rightarrow} \pi_{*} \tilde{\mathcal{N}} \rightarrow R^{1} \pi_{*} \tilde{\mathcal{V}} \rightarrow \ldots
$$

If we assume that the vector bundle is semistable over every elliptic fiber (this need not always be true, as we will see; it is necessary only that the vector bundle be semistable over generic elliptic fibers), then $R^{1} \pi_{*} \tilde{\mathcal{V}}$ is identically zero because its presheaf is locally of the form $H^{1}\left(E, \mathcal{O}\left(p_{i}\right)\right)$. Now consider the action of the right exact functor $\pi^{*}$ over the last sequence. Since the elliptic fibration map $\pi$ is a flat morphism, it does not have higher left derived functors. [We have $\operatorname{Tor}_{1 S}(M, R)=0$ due to the flatness, where $S$ is the ring that corresponds to $\mathcal{O}_{B}, R$ corresponds to $\mathcal{O}_{X}$, and $M$ is the free module corresponding to $\mathcal{V}$ (see, e.g., Chap. 3 in [34]).] So we get

$$
0 \rightarrow \pi^{*} \pi_{*} \tilde{\mathcal{V}} \rightarrow \pi^{*} \pi_{*} \tilde{\mathcal{H}} \stackrel{\bar{F}}{\rightarrow} \pi^{*} \pi_{*} \tilde{\mathcal{N}} \rightarrow 0
$$

Note that $\pi^{*} \pi_{*} \tilde{\mathcal{V}}$ is a vector bundle whose fibers over a point $p$ are generated by the global sections of $\tilde{\mathcal{V}}$ over elliptic curve $E_{b}$, where $b=\pi(p)$. So Eq. (5.6) tells us that 
if we find the global sections of $\tilde{\mathcal{H}}$ and $\tilde{\mathcal{N}}$, then the kernel of the induced map $F$ is isomorphic to $\pi^{*} \pi_{*} \tilde{\mathcal{V}}$. So the locus is where the rank of the kernel drop coincides with the spectral cover. To clarify these rather abstract ideas, in the following subsection, we explicitly compute the spectral cover of two examples which will be used in the final subsection.

\section{B. Examples}

To begin, we assume that the $K 3$ manifold can be written in the following simple toric form,

\begin{tabular}{|ccccc|c|}
\hline \multicolumn{5}{c|}{$x_{i}$} & $\Gamma^{j}$ \\
\hline 3 & 2 & 1 & 0 & 0 & -6 \\
6 & 4 & 0 & 1 & 1 & -12 \\
\hline
\end{tabular}

$$
\left[\begin{array}{c}
x-z^{2} \frac{-f_{2} h_{4}+d f_{6}}{c f_{2}} \\
0 \\
-\frac{b}{c} y-x z \frac{f_{2} g_{2}-b f_{4}}{c f_{2}}-z^{3} \frac{f_{2} g_{6}-d f_{8}}{c f_{2}} \\
-x^{2} \frac{f_{4}}{f_{2}}+y z \frac{b f_{6}}{c f_{2}}-x z^{2} \frac{c f_{8}+f_{4} h_{4}-g_{2} f_{6}}{c f_{2}}-z^{4} \frac{f_{8} h_{4}-f_{6} g_{6}}{c f_{2}}
\end{array}\right.
$$$$
\left.\begin{array}{c}
0 \\
x \frac{c f_{2}}{f_{2} h_{4}-d f_{6}}+z^{2} \\
-z^{3} \frac{f_{2} h_{2}-d g_{4}}{f_{2} h_{4}-d f_{6}}+-x z \frac{a d}{d f_{6}-f_{2} h_{4}} \\
x^{2} \frac{a c}{d f_{6}-f_{2} h_{4}}-x z^{2} \frac{c g_{4}+a h_{4}}{f_{2} h_{4}-d f_{6}}-z^{4} \frac{-f_{6} h_{2}+g_{4} h_{4}}{f_{2} h_{4}-d f_{6}}
\end{array}\right],
$$

Example 1: The first example is the following $S U(2)$ monad,

\begin{tabular}{|llll|ll|}
\hline \multicolumn{4}{c|}{$\Lambda$} & \multicolumn{2}{c|}{$p$} \\
\hline 1 & 1 & 2 & 3 & -3 & -4 \\
1 & 5 & 3 & 7 & -9 & -7 \\
\hline
\end{tabular}

The second Chern class of this monad is 12 . The map $F$ of the monad is given by the following generic matrix,

$F \sim\left[\begin{array}{cccc}x f_{4}+z^{2} f_{8} & a x+z^{2} g_{4} & z f_{6} & f_{2} \\ b y+x z g_{2}+z^{3} g_{6} & z^{3} h_{2} & c x+z^{2} h_{4} & d z\end{array}\right]$,

where subscripts indicate the degree of homogeneous polynomials over $\mathbb{P}^{1}$. With this choice, it can be verified that the kernel of $\bar{F}$ in Eq. (5.6) takes the following generic form, where $f_{i}, g_{i}, h_{i}$ are polynomials in terms of base coordinates with degree $i$, and $a, b, c, d$ are constants. The common factor of the minors of Eq. (5.10) is

$$
c f_{2} x+\left(f_{2} h_{4}-d f_{6}\right) z^{2} \text {. }
$$

From the previous discussion, we might naively conclude that Eq. (5.11) must be the spectral cover. However, in the Fourier-Mukai discussion, it was noted that the divisor class of the spectral cover should be $2 \sigma+12 D$. So in the expression above, we are clearly missing a degree 6 polynomial in Eq. (5.11). The correct spectral cover should be

$$
S=F_{6}\left(c f_{2} x+\left(f_{2} h_{4}-d f_{6}\right) z^{2}\right) .
$$

But why then is $F_{6}$ missing? The reason is that, in the previous subsection, we assumed that the vector bundles are semistable over every fiber. This not necessarily true. It is possible to start from a stable bundle and modify it in a way that it becomes semistable over every fiber [46].

To see clearly what happens, let us first find the elliptic fibers such that the vector bundle over them is unstable. Note that from Eq. (5.11) it can be seen that the spectral cover is a nondegenerate two-sheeted surface and that, for a generic $E,\left.V\right|_{E}=\mathcal{O}(p-\sigma) \oplus \mathcal{O}(q-\sigma)$, where $p+q=2 \sigma$. So $\left.V\right|_{E}$ does not have a global section over almost any fiber except when $p=q=\sigma$. These points are on the intersection of $z=0$ and the spectral cover, which are the zeros of $f_{2} F_{6}$. The idea then is to see if we can find the elliptic fibers which, over the vector bundle (not its twisted descendant), can have a global section. So all we need to do to find $F_{6}$ is to study the kernel of the induced map in the following sequence:

$$
0 \rightarrow \pi_{*} \mathcal{V} \rightarrow \pi_{*} \mathcal{H} \stackrel{F_{\text {ind }}}{\rightarrow} \pi_{*} \mathcal{N} \rightarrow R^{1} \pi_{*} \mathcal{V} \rightarrow \ldots
$$

where the induced map $F_{\text {ind }}$ in the above case is a $7 \times 7$ matrix in terms of the base coordinates. Generically its rank is 7 except over the zeros of $f_{2} F_{6}$, so we can read the missing polynomial from this form. Please note that the above computations are local, globally $\pi_{*} \mathcal{V}=0$. Because $\mathcal{V}$ is locally free and $\pi$ is a flat morphism, the pushforward of $\mathcal{V}$ should also be torsion-free (see Chap. III. 9.2 of [33]).

Interestingly, when we repeat the same analysis after twisting with $\mathcal{O}(\sigma)$ and $\mathcal{O}(2 \sigma)$, the rank of the corresponding induced map drops over $F_{6}$ and nowhere, respectively. This means that the bundle over those fibers takes the following form,

$$
\left.\mathcal{V}\right|_{E_{\text {missing }}}=\mathcal{O}(p) \oplus \mathcal{O}(-p)
$$


therefore $h^{0}(E, \mathcal{V} \otimes \mathcal{O}(\sigma)) \geq 2$, and the rank of the kernel in (5.6) (which generically is the same as the rank of the bundle-in this case, 2) does not drop over the points of these fibers. So the algorithm suggested in [45] does not find them. But it can be seen from Eq. (4.5) that these (whole) elliptic fibers are in support of the spectral sheaf. In summary, a detailed analysis along the lines sketched above shows that the missing component is given by

$$
F_{6}=h_{2} c f_{2}^{2}-c d f_{2} g_{4}+a d f_{2} h_{4}-a d^{2} f_{6} .
$$

where each element in the expression polynomial above is defined using the monad map in Eq. (5.9).

Example 2: The second example is interesting because its spectral cover is degenerate (in this case, a nonreduced scheme):

\begin{tabular}{|llll|ll|}
\hline \multicolumn{3}{c|}{$\Lambda$} & \multicolumn{2}{|c|}{$p$} \\
\hline 0 & 1 & 2 & 3 & -2 & -4 \\
2 & 1 & 3 & 7 & -6 & -7 \\
\hline
\end{tabular}

The second Chern class of this monad is $c_{2}(V)=6$, so from the previous discussion, it is clear that the divisor class of its spectral cover must be $2 \sigma+6 D$. The number of global sections of $\mathcal{H}$ and $\mathcal{N}$ is 7 and 6, respectively, which tells us that the kernel of $F_{\text {ind }}$ is at least one dimensional over almost every elliptic fiber. Since $\mathcal{V}$ is a stable, rank 2 bundle with $c_{1}(\mathcal{V})=0$, we conclude that either $\left.\mathcal{V}\right|_{E}=\mathcal{O} \oplus \mathcal{O}$ or $\left.\mathcal{V}\right|_{E}=\epsilon_{2}{ }^{9}$. In both cases, the spectral cover must have the following general form,

$$
S=F_{6} z^{2}
$$

In fact, by computing the $F_{\text {ind }}$ directly, we can see the rank of the kernel is always 1 , so $\left.\mathcal{V}\right|_{E}=\epsilon_{2}$. As before, we can compute the kernel of Eq. (5.6), to generate the spectral cover,

$$
\left[\begin{array}{cc}
z & 0 \\
0 & z^{2} \\
-\frac{x z}{f_{3}}-z^{3} \frac{f_{4}}{f_{3}} & z^{3} \frac{f_{5}}{f_{3}} \\
a \frac{x^{2}}{f_{3}}+x z^{2} \frac{f_{4}}{f_{3}}+z^{4} \frac{f_{8}}{f_{3}} & b y z+x z^{2} \frac{g_{5}}{f_{3}}+z^{4} \frac{f_{9}}{f_{3}}
\end{array}\right]
$$

Then we look at the minors, and the common factor should be spectral cover. However, as in the previous examples, the algorithm in [45] misses the polynomial $F_{6}$. The reason is similar to the previous example: The bundle is unstable over the zeros of $F_{6}$. It can be shown that the correct spectral cover is indeed

\footnotetext{
$\left.{ }^{9} \mathrm{By} \mathcal{V}\right|_{E}=\epsilon_{2}$, we mean the unique nontrivial extension of the trivial bundles over the elliptic curve.
}

$$
S=\left(F_{3}\right)^{2} z^{2}
$$

where $F_{3}$ is the entry $(1,3)$ of the monad's map [i.e., the map between the line bundles $\mathcal{O}_{X}(2,3)$ and $\left.\mathcal{O}_{X}(2,6)\right]{ }^{10}$

\section{Counterexamples of the conjecture}

Here we return to the main goal of this section. Suppose that we have two target space dual GLSMs that describe different stable bundles over elliptic $K 3$ surfaces. The goal is to check whether their F-theory dual geometries can be related via a change in $K 3$ fibrations (i.e., a change in $\mathbb{P}^{1}$ fibrations in the twofold base of the CY threefold). Generally the base of the F-theory threefold will be a Hirzebruch surface $F_{n}$, where $n$ is given by the twist in Eq. (4.12). The only situation that can accommodate such multiple fibration structures is when $n=0$. So here we focus on this case and demand that the second Chern class of both heterotic vector bundles be 12 .

We assume that one of the target space dual geometries is given by monads on the toric $K 3$ (5.7). We also write the elliptically fibered $K 3$ in Weierstrass form,

$$
y^{2}=x^{3}+f_{8}\left(x_{1}, x_{2}\right) x z^{4}+f_{12}\left(x_{1}, x_{2}\right) z^{6},
$$

where $x_{1}$ and $x_{2}$ are the coordinates of the base $\mathbb{P}^{1}$. To find explicit examples for target space duality, recall there are several constraints that must be met. First, it is necessary to have a well-defined GLSM. This means that the first Chern class of both bundles should be zero, and the second Chern class of both bundles (or sheaves) should be 12 .

In addition, we must make sure that the hybrid phase in which we do the TSD "exchange" of $G$ and $F$ actually exists. In the process of generating the TSD pairs, it may happen that singularities arise in the bundle or manifold (we expect that crepant resolutions should exist for the manifold and that the singularities in the "bundle" should be codimension 2 in the base so that the sheaf is torsionfree). In addition to these constraints for the GLSM, there is another practical requirement for finding the F-theory geometry: We prefer to work with $S U(N)$ bundles which have a nondegenerate spectral cover. If this requirement is not satisfied, it is still possible to find the F-theory dual, but we should remember that the form of the spectral sheaves can be vastly more rich/complex in these cases. This enhanced data in the Picard group will not be manifest in the spectral cover, or in the complex structure moduli of the dual F-theory geometry. Instead, it will be related in the

\footnotetext{
${ }^{10}$ After a thorough computation, we can show that

$$
0 \rightarrow \mathcal{J} \rightarrow F M^{1}(V) \rightarrow \mathcal{O}_{\sigma} \rightarrow 0
$$

where $\mathcal{J}$ is a torsion sheaf supported over $\{z=0\} \cup\left\{F_{3}=0\right\}$, and its rank over $\left\{F_{3}=0\right\}$ is 2 . $\mathcal{J}$ can be computed explicitly,
} but doing so is outside of the scope of this paper. 
dual F-theory to nilpotent Higgs bundles over singular curves [16,47-49].

It is straightforward to find many GLSMs where at least one of the bundles (say, $\mathcal{V}_{1}$ ) has $S U(N)$ structure, and where spectral cover is nondegenerate; for example, consider Eq. (5.8) once again,

\begin{tabular}{|llll|ll|}
\hline \multicolumn{4}{|c|}{$\Lambda$} & \multicolumn{2}{|c|}{$p$} \\
\hline 1 & 1 & 2 & 3 & -3 & -4 \\
1 & 5 & 3 & 7 & -9 & -7 \\
\hline
\end{tabular}

with Chern class

$$
C_{2}\left(V_{1}\right)=5 \sigma^{2}+22 \sigma D+23 D^{2}=12 .
$$

It should be noted that the algorithm for determining the spectral cover, using the methods of [45], was sketched above, but when the spectral cover becomes reducible (which can still be reduced), it is not guaranteed that those methods would find the full spectral cover [i.e., usually some (vertical) components will be missed]. One can find these components by closer examination of the morphisms that define the bundle and elliptic fibration, as we saw in the last subsection. The spectral cover (schematically) is then given by Eq. (5.12),

$$
S=F_{6}\left(f_{2} x+f_{6} z^{2}\right) .
$$

Note that Eq. (5.8) by itself is not a well-defined linear sigma model; therefore we need another bundle such that its structure group is embedded in the other $E_{8}$ factor. This second bundle must also have a GLSM description over the same $K 3$, and its second Chern class should be

$$
C_{2}\left(V_{2}\right)=6 \sigma^{2}+24 \sigma D+21 D^{2}=12 .
$$

Since the existence of this bundle with above properties may not be quite obvious, we turn now to constructing appropriate examples explicitly.

\section{Example 1}

We can construct an example $\mathcal{V}_{2}$ (though not the most general such bundle) as a direct sum of two bundles, each defined by the monad in Eq. (5.16) (which we denote here by $\left.\mathcal{V}_{0}\right)$, with $c_{2}\left(\mathcal{V}_{0}\right)=6$ :

$$
\mathcal{V}_{2}=\mathcal{V}_{0} \oplus \mathcal{V}_{0}
$$

For this monad bundle, the spectral cover was found to be of the form given in Eq. (5.19). In addition, the rank 1 sheaf on the spectral cover can be readily constrained. Here $F M^{0}\left(\mathcal{V}_{0}\right)$ is zero by results in [33] (see Sec. III. 12, the final theorem). So we actually have the following short exact sequence,

$$
F M^{1}\left(\mathcal{V}_{2}\right)=F M^{1}\left(\mathcal{V}_{0}\right) \oplus F M^{1}\left(\mathcal{V}_{0}\right)
$$

where the support of $F M^{1}\left(\mathcal{V}_{2}\right)$, which is the spectral cover of $\mathcal{V}_{2}$, is the union of the spectral covers associated with the two copies of $\mathcal{V}_{0}$. The resulting spectral cover is a nonreduced scheme, which can be realized using the following polynomial $^{11}$ :

$$
S\left(V_{2}\right)=\left(F_{3}\right)^{2}\left(G_{3}\right)^{2} z^{4} .
$$

Before turning to the F-theory dual of this geometry, let us first construct a target space dual model for the above GLSM. To do that we add new chiral fields in a way that, after integrating them out, we return to the initial model. This can done by adding "repeated entries" to the charge matrix of the $K 3$, and it can lead to multiple TSD geometries (all still of the same topological type of manifold and bundle, of course). One possibility is

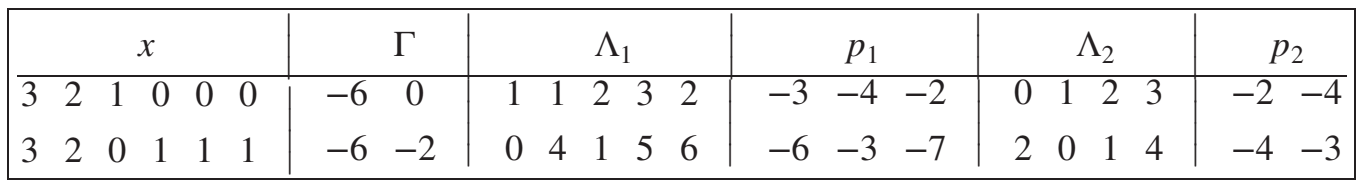

This heterotic geometry ( $K 3$ manifold and bundle) has pointlike singularities in the would-be bundle- that is, it is a rank 2 torsion-free sheaf rather that a vector bundle [50].

\footnotetext{
${ }^{11}$ Generally the two $\mathcal{V}_{0}$ 's in the above construction can be related by a continuous deformation, so we consider $F_{3}$ and $G_{3}$ to be different generic degree 3 polynomials.
}

With this pair of TSD bundles over $K 3$ in hand, we are now in a position to consider the dual F-theory geometry. In this case, we will ask the key question: Are the two GLSMs/geometries, i.e., those defined by $\mathcal{V}_{1}$ and $\mathcal{V}_{2}$ and its TSD dual in Eq. (5.26), realized as different fibrations of a single F-theory geometry? 
By the results of the previous subsection, the complex structure of the Calabi-Yau threefold can be readily determined:

$$
\begin{aligned}
Y^{2}= & X^{3}+F\left(u_{1}, u_{2}, x_{1}, x_{2}\right) X Z^{4} \\
& +G\left(u_{1}, u_{2}, x_{1}, x_{2}\right) z^{6}, \\
F\left(u_{1}, u_{2}, x_{1}, x_{2}\right)= & u_{1}^{4} u_{2}^{4} f_{8}\left(x_{1}, x_{2}\right) \\
& +u_{1}^{3} u_{2}^{5} F_{6}\left(x_{1}, x_{2}\right) f_{2}\left(x_{1}, x_{2}\right), \\
G\left(u_{1}, u_{2}, x_{1}, x_{2}\right)= & u_{1}^{7} u_{2}^{5}\left(F_{3}\left(x_{1}, x_{2}\right)\right)^{2}\left(G_{3}\left(x_{1}, x_{2}\right)\right)^{2} \\
& +u_{1}^{6} u_{2}^{6} f_{12}\left(x_{1}, x_{2}\right) \\
& +u_{1}^{5} u_{2}^{7} F_{6}\left(x_{1}, x_{2}\right) f_{6}\left(x_{1}, x_{2}\right) .
\end{aligned}
$$

As frequently happens with degenerate spectral data, we find that the apparent F-theory gauge symmetry seems to be in contradiction with what is expected from the heterotic theory we have engineered. By inspection of the discriminant of Eq. (5.27), it is straightforward to see that there appears to be an $E_{7}$ symmetry on $u_{1} \rightarrow 0$, and an apparent $E_{8}$ singularity above the curve $u_{1} \rightarrow \infty$. This might seem to be in contradiction with the expected gauge symmetry of $S O(12)$ in the hidden sector [determined as the commutant of the $S U(2) \times S U(2)$ structure group defined by the reducible bundle in Eq. (5.23)]. However, in the case of degenerate spectral covers, we naturally expect that T-brane-type solutions $[16,47]$ may well arise in the dual F-theory geometry. That is, we expect a nilpotent $S U(2) \times S U(2)$ Higgs bundle over the 7-brane which wraps around this curve $\left(u_{2}=0\right)$ and breaks the spacetime gauge group to $S O(12)$ as expected (see [17] for a similar construction).

Next, as demonstrated in [43], changing the $K 3$ (and $\mathbb{P}^{1}$, respectively) fibration in the F-theory geometry simply amounts to exchanging the vertical $\mathbb{P}^{1}$ (whose coordinates are $u_{1}$ and $u_{2}$ ) with the horizontal $\mathbb{P}^{1}$, which is the base in the initial heterotic $K 3$ surface in Eq. (5.7). This means that the vertical $\mathbb{P}^{1}$ becomes the base of a dual heterotic $K 3$ surface. To determine gauge groups in the dual heterotic theory, the discriminant curve must be considered. This is shown in Fig. 1. The figure on the right-hand side shows the discriminant of the F-theory Calabi-Yau threefold. The line at the top is the locus of the $E_{7}$ singularity, and the one at the bottom corresponds to $E_{8}$. The curve is the locus of the $I_{1}$ singularities. It intersects eight times with the $I I I^{*}$ curve, where on six of them it has triple point singularities. These six points are exactly the zeros of $F_{6}$ in Fig. 1 , which naively correspond to pointlike instantons that are responsible for the vertical components in spectral cover of $\mathcal{V}_{1}$ (not taking into account the spectral sheaf/T-brane data). Similarly, the $I_{1}$ curve intersects with $I I^{*}$ at two sets of three points, which are the zeros of $F_{3}$ and $G_{3}$ in Eq. (5.25), and over all of them, the curve has double point singularities.

We can expand the polynomials in Eq. (5.27) in terms of $x_{1}$ and $x_{2}$ and read the dual heterotic complex structure from there. Clearly we see that the elliptic $K 3$ in the new heterotic dual must be singular. In particular, it exhibits singular $E_{8}$ and $E_{7}$ located at $u_{1}=0$ and $u_{1} \rightarrow \infty$, respectively (with an expected instanton number of 12 on each locus). This is a highly nonperturbative limit of the string theory. This exchange of gauge symmetry with singularities in the base $K 3$ surface arising in the heterotic theory seems to be a generic feature of exchanging F-theory fibrations [27]. As a result, it seems impossible to get something which is purely smooth/perturbative on both sides like Eq. (5.26) from such a change of fibration. This shows that at least some of the TSD dual pairs cannot be seen simply as different fibrations of the F-theory geometry. We explore these possibilities a little more in one further example.

\section{E. Example 2}

In this example, the starting geometry/bundles are the same as before, but here we present another TSD geometry that can also be described easily by spectral cover. So once again we take as our starting point the manifold/bundle

\begin{tabular}{|ccccc|c||rrrr|rr|}
\hline \multicolumn{4}{c|}{$x_{i}$} & \multicolumn{1}{|c||}{$\Gamma^{j}$} & \multicolumn{4}{c||}{$\Lambda^{a}$} & \multicolumn{2}{|c|}{$p_{l}$} \\
\hline 3 & 2 & 1 & 0 & 0 & -6 & 1 & 1 & 2 & 3 & -3 & -4 \\
6 & 4 & 0 & 1 & 1 & -12 & 1 & 5 & 3 & 7 & -9 & -7 \\
\hline
\end{tabular}

and embed it into a larger GLSM by adding a new gauge field, and fermionic and chiral fields:

\begin{tabular}{|cccccccc|cc||ccc||cccccc|cccc|}
\hline \multicolumn{1}{c|}{$x_{i}$} & \multicolumn{4}{|c||}{$\Gamma^{j}$} & \multicolumn{1}{|c|}{$\Lambda^{a}$} & \multicolumn{4}{|c|}{$p_{l}$} \\
\hline 3 & 2 & 1 & 0 & 0 & 0 & 0 & 0 & -6 & 0 & 0 & 1 & 1 & 2 & 3 & 3 & 3 & -3 & -4 & -3 & -3 \\
6 & 4 & 0 & 1 & 1 & 0 & 0 & 1 & -12 & 0 & -1 & 1 & 5 & 3 & 7 & 8 & 9 & -9 & -7 & -8 & -9 \\
0 & 0 & 0 & 0 & 0 & 1 & 1 & 0 & 0 & -1 & 0 & 0 & 0 & 0 & 0 & 0 & 0 & -1 & 0 & 0 & 0 \\
\hline
\end{tabular}

After performing the combinatoric exchange (i.e., the usual TSD procedure), this yields the new TSD geometry 


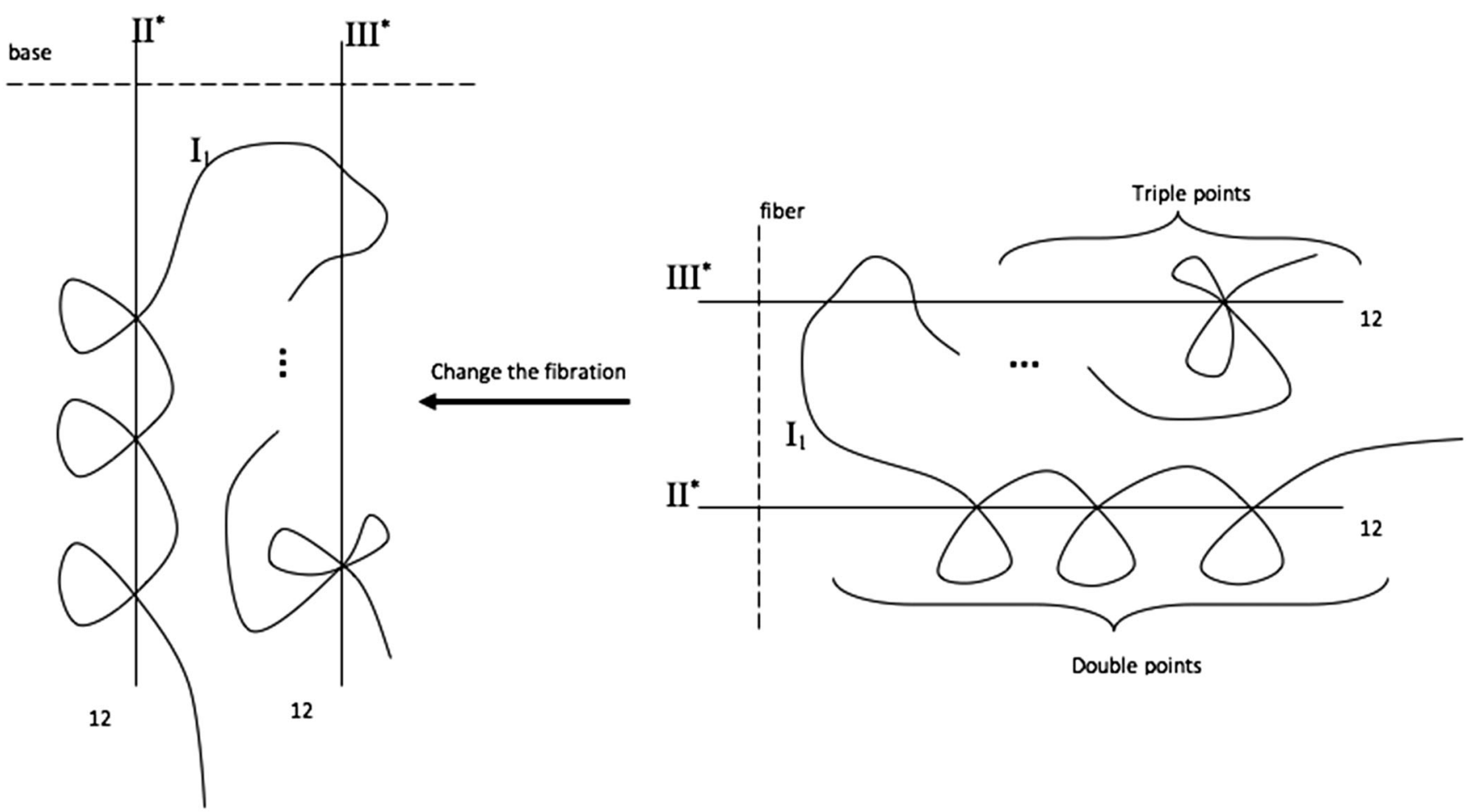

FIG. 1. The vertical dotted line on the right-hand side is the "vertical $\mathbb{P}^{1}$ ". After change of fibration on the left-hand side, the same $\mathbb{P}^{1}$ will be the base of the dual heterotic $K 3$.

\begin{tabular}{|cccccccc||ccc||rcccc|ccc|}
\hline \multicolumn{1}{c|}{$x_{i}$} & \multicolumn{4}{|c||}{$\Gamma^{j}$} & \multicolumn{4}{|c||}{$\Lambda^{a}$} & \multicolumn{4}{|c|}{$p_{l}$} \\
\hline 3 & 2 & 1 & 0 & 0 & 0 & 0 & 0 & -6 & 0 & 0 & 1 & 1 & 2 & 3 & 3 & -3 & -4 & -3 \\
6 & 4 & 0 & 1 & 1 & 0 & 0 & 1 & -12 & -1 & 0 & 5 & 3 & 7 & 8 & -9 & -7 & -8 \\
0 & 0 & 0 & 0 & 0 & 1 & 1 & 0 & 0 & -1 & -1 & 0 & 0 & 0 & 0 & 1 & -1 & 0 & 0 \\
\hline
\end{tabular}

The advantage of this new example is that it is possible to compute the spectral cover of both sides easily, ${ }^{12}$ and they are both reducible but still reduced:

$$
\begin{aligned}
& S_{1}=F_{6}\left(f_{2} X+f_{6} Z^{2}\right), \\
& S_{2}=F_{7}\left(f_{1} X+f_{5} Z^{2}\right) .
\end{aligned}
$$

As in the previous example, we can readily construct the F-theory geometry of both sides and check whether or not they are related by exchanging the fibration. The Weierstrass polynomials, $F$ and $G$, of the dual F-theories is given by

\footnotetext{
${ }^{12}$ In the previous example, the base $\mathbb{P}^{1}$ was defined as a conic inside $\mathbb{P}^{2}$. However, the spectral cover equations would be in terms of the ambient space coordinates, and imposing nonlinear relations between the coordinates to define the $\mathbb{P}^{1}$ makes the situation somewhat obscure.
}

$$
F_{1}=\mathcal{O}\left(u_{1}^{5}\right)+u_{1}^{4} u_{2}^{4} f_{1}^{8}\left(v_{1}, v_{2}\right)+u_{1}^{3} u_{2}^{5} F_{6}\left(v_{1}, v_{2}\right) f_{2}\left(v_{1}, v_{2}\right),
$$

$G_{1}=\mathcal{O}\left(u_{1}^{7}\right)+u_{1}^{6} u_{2}^{6} g_{1}^{12}\left(v_{1}, v_{2}\right)+u_{1}^{5} u_{2}^{7} F_{6}\left(v_{1}, v_{2}\right) f_{6}\left(v_{1}, v_{2}\right)$,

$$
F_{2}=\mathcal{O}\left(v_{1}^{5}\right)+v_{1}^{4} v_{2}^{4} f_{2}^{8}\left(u_{1}, u_{2}\right)+v_{1}^{3} v_{2}^{5} F_{7}\left(u_{1}, u_{2}\right) f_{1}\left(u_{1}, u_{2}\right),
$$

$G_{2}=\mathcal{O}\left(v_{1}^{7}\right)+v_{1}^{6} v_{2}^{6} g_{2}^{12}\left(u_{1}, u_{2}\right)+v_{1}^{5} v_{2}^{7} F_{7}\left(u_{1}, u_{2}\right) f_{5}\left(u_{1}, u_{2}\right)$.

As in the previous example, the change in fibration can be realized in $F_{1}$ and $G_{1}$ by reexpanding these polynomials in terms of $v_{1}$ and $v_{2}$. Then if the dual (F-theory) geometries are related through changing the fibration, after this rearrangement, $F_{1}$ and $G_{1}$ must be equal to $F_{2}$ and $G_{2}$. 
But since $F_{2}$ and $G_{2}$ have an order 3 zero at $v_{1}=0$, it means that $f_{1}^{8}\left(v_{1}, v_{2}\right)$ and $g_{1}^{12}\left(v_{1}, v_{2}\right)$ must have an order 3 zero at $v_{1}=0$. Recall that these two polynomials are the $f$ and $g$ of the dual heterotic $K 3$ surface, so the above argument tells us if the TSD geometries are related to different fibrations of the same geometry in F-theory, both TSD Calabi-Yau twofolds must have an $E_{7}$ singularity at some point on the base. Thus, once again, we see that the exchange of fibration leads to a perturbative/nonperturbative duality in heterotic theories [43] and not the apparent correspondence arising from TSD.

In summary, if we start with two perfectly smooth TSD geometries, they cannot be related through different $K 3$ fibrations of a single F-theory threefold. But if we allow both $K 3$ surfaces to be singular and at the same time put bundles/small instantons over them, they might be dual to a single geometry in F-theory. ${ }^{13}$

Having determined that the multiple fibrations are not describing the TSD exchange in six dimensions, we can take a step back and ask, what $F$-theory correspondence is induced by TSD in six dimensions? Since the spectral covers in Eqs. (5.31) and (5.32) are relatively simple, we can try to roughly figure out some generalities about the Ftheory duals of each of them. Let us start with the first one. The topology of the vector bundle fixes the dimension of the moduli space of the bundle,

$$
h^{1}\left(V_{1} \otimes V_{1}^{*}\right)=42 .
$$

We can describe them in terms of the spectral data as follows,

$$
\begin{aligned}
\operatorname{dim}\left(\mathcal{M}_{V}\right)= & \operatorname{dim}(\operatorname{cplx}(C))+\operatorname{dim}(\operatorname{Jac}(C))+6 p t s \\
& +6 \times \operatorname{dim}(\operatorname{Jac}(E))+\text { gluing }
\end{aligned}
$$

where $C$ is the irreducible smooth curve defined by $f_{2} X+f_{6} Z^{2}$. By "6pts" we mean the d.o.f. in choosing the location of the six points defined by the zero set of $F_{6}=0$, and over them we have six elliptic curves (whose Jacobians must also be taken into account), and finally, "gluing" denotes the d.o.f. associated with the choice of spectral sheaf at the intersection of the six vertical fibers with $C$. The genus of $C$ can be computed easily,

$$
g(C)=9 .
$$

Therefore the dimension of the Jacobian and the complex structure of $C$ must be 9 . On the other hand, obviously, Jacobian of $E$ is one dimensional, and the contribution of the gluing is 12 dimensional (each vertical fiber intersects $C$

\footnotetext{
${ }^{13}$ But we should recall that the GLSM is only a perturbative formulation and clearly lacks information on the full string theory in such a context.
}

at two points). Therefore the total dimension of the Moduli space is

$$
\operatorname{dim}\left(\mathcal{M}_{V}\right)=9+9+6+6+12=42
$$

Now to obtain the F-theory EFT, we must use the spectral data as explained before and infer the form of the complex structure of the CY threefold. From this procedure, it can be seen that there are $6(4,6,12)$ points in the F-theory geometry. Since the heterotic dual is a perturbative model, we should consider these singularities as the singular limit of the following deformations,

$$
\begin{aligned}
F_{1}= & \mathcal{O}\left(u_{1}^{5}\right)+u_{1}^{4} u_{2}^{4} f_{1}^{8}\left(v_{1}, v_{2}\right) \\
& +u_{1}^{3} u_{2}^{5}\left(F_{6}\left(v_{1}, v_{2}\right) f_{2}\left(v_{1}, v_{2}\right)+\epsilon F_{8}\left(v_{1}, v_{2}\right)\right), \\
G_{1}= & \mathcal{O}\left(u_{1}^{7}\right)+u_{1}^{6} u_{2}^{6} g_{1}^{12}\left(v_{1}, v_{2}\right) \\
& +u_{1}^{5} u_{2}^{7}\left(F_{6}\left(v_{1}, v_{2}\right) f_{6}\left(v_{1}, v_{2}\right)+\lambda F_{12}\left(v_{1}, v_{2}\right)\right),
\end{aligned}
$$

where $\epsilon$ and $\lambda$ correspond to deforming the Higgs field over the 7-branes [16,51]. Therefore we can deform these two theories into each other by continuously deforming the Higgs bundle. This reflects the fact that the moduli space of the vector bundles on $K 3$ is connected. Phrased differently, the existence of apparent $(4,6,12)$ points in the putative dual F-theory indicates that such solutions can only be dual to the expected perturbative heterotic theories in the case that T-brane solutions arise. This has been seen before in [47] and is a substantial hint that G-flux must play an important role in the nontrivial F-theory correspondence expected in four-dimensional compactifications.

It is worth commenting briefly also on another branch of the theory visible from this singular limit. We can increase the number of tensor multiplets in the six-dimensional Yang-Mills (YM) theory by performing small instanton transitions (i.e., moving NS5-/M5- branes off the $E_{8}$ fixed plane in the language of heterotic M-theory). For bundles described as spectral covers, this small instanton limit is visible with the spectral cover becoming reducible and vertical components (corresponding to small instantons) appearing (note that this limit must also set all gluing data to zero). Naively, it seems that this limit appears to be different for the TSD pair of bundles defined by Eqs. (5.31) and (5.32) since they exhibit different degree polynomials defining their vertical components (i.e., $F_{6}$ vs $F_{7}$ ). However, this is simply a statement that the mapping of moduli in this case may exchange what are spectral cover deformations in one description with data associated with the Jacobian of the spectral cover (i.e., gluing data in this singular case). To really obtain the same point in moduli space, we must consider a scenario in which both halves of the TSD gain the same number of tensor multiplets (i.e., we pull either six or seven 5-branes into the bulk). In this case, it would be intriguing to analyze the dual F-theory 
geometry-which would correspond to blowing up the base of the elliptic fibration. We expect in this case that the F-theory threefold will still be $K 3$ fibered but no longer of such a simple form. In particular, the elliptic fibration over a Hirzebruch surface would be modified to become a more general conic bundle [27]. We will return to questions of a similar geometric nature in the following section.

Let us briefly summarize the results of our sixdimensional investigation. We have seen that after exchanging $K 3$ fibrations within the F-theory geometry, the dual heterotic $K 3$ surface must become singular, and therefore perturbative smooth heterotic geometries arising in TSD pairs cannot, in general, be realized as different fibrations within F-theory. On the other hand, we saw that the dual F-theory EFTs arising from the chosen TSD pairs must crucially rely on data from the intermediate Jacobian of the CY threefold-so-called T-brane solutions-in order to give rise to the same physical theories. Starting from such points, we can deform back to smooth points in the CY threefold moduli space and identify the theories. Any possible correspondences within the tensor branch of the six-dimensional theories must involve more complicated $K 3$ fibrations (i.e., conic bundles), and we leave this exploration to future work.

\section{F-THEORY DUALS OF FOUR-DIMENSIONAL HETEROTIC TSD PAIRS}

In Sec. III, we provided a nontrivial example of a heterotic TSD pair in which both $X$ and $\tilde{X}$ were elliptically fibered. It is now natural to ask, what are the F-theory duals of these heterotic theories? As we will explain below, this example (and others like it that we have found) seem to force beyond the arena of "standard" heterotic/F-theory duality (as in the canonical reference [12]) by including several important features in the dual geometries. In this section, we will not try to solve all of the obstacles that arise at once. Instead, we will outline what can be determined about the dual F-theory geometries and where new tools will be needed to fully probe this correspondence. Many of these we are currently developing [13,14], and we hope to definitively answer these questions in future work.

As a first step toward determining the dual F-theory geometry, the data of the heterotic bundle must be taken through a Fourier-Mukai transform to be presented as spectral data (see the discussion in Sec. V). However, in this, we immediately encounter several problems. The first of these is that, unlike in the case of heterotic/F-theory dual pairs studied in the literature to date, neither of these heterotic CY elliptic threefolds is in Weierstrass form.

To be specific, we focus on the examples in Sec. III (though similar obstacles will arise, in general, in this context). Recall that each of the CY threefolds listed in Eq. (3.14) admitted two rational sections. Those for $X$ in Eq. (3.14) lie in the following classes,
$\left[\sigma_{1}(X)\right]=-D_{1}+D_{2}+D_{3}, \quad\left[\sigma_{2}(X)\right]=2 D_{1}-D_{2}+5 D_{3}$.

where $D_{i}$ represent a basis of divisors on $X$ (inherited from the ambient space hyperplanes by restriction). By "rational", it is meant that these divisors are isomorphic to blowups of the base manifold (in this case, $P^{2}$ ). The first difficulty with this example is that the standard FourierMukai transformation with Poincare bundle is not applicable here. The reason for this is that we need the zero section to intersect at exactly one point on every fiber, but both of the sections described above wrap around a finite number of rational curves (which are components of reducible fibers). We have shown [13] that in specific situations one can use flop transitions to make one of the sections holomorphic, and since derived categories are invariant under the flop transitions (i.e., there is a specific Fourier-Mukai functor for flops), it is still possible to define the spectral data in the "flopped" geometry. However, the example given in Eq. (3.14) proves to be too complicated to be analyzed in this manner since $\sigma_{1}$ and $\sigma_{2}$ wrap around 27 and 127 rational curves, respectively, rendering the necessary birational transformations (i.e., flops) impractical.

In principle, one might hope to bypass this difficulty by transitioning $X$ directly to its Weierstrass form (by blowing down the reducible components of fibers), following the Deligne procedure outlined in [26,27]. However, this poses difficulties in a heterotic theory in that it is unclear how the heterotic bundle data should be appropriately mapped to this singular limit of $X$.

Nonetheless, if we choose $\sigma_{1}$ as the zero section, it can be demonstrated that the spectral cover in the singular Weierstrass limit has the same divisor class as before (this is seen by taking the FM transform before blowing down the reducible fiber components). In other words, if we write the second Chern class as

$$
c_{2}(V)=36 \sigma_{1} H+14 S_{s h} H+156 f,
$$

where $H$ is the (pullback of the) hyperplane divisor in the base, $S_{s h}$ is the divisor corresponding to the Shioda map [52-54] for nontrivial Mordell-Weil group

$$
S_{s h}=\sigma_{2}-\sigma_{1}-18 H,
$$

and $f$ is the fiber class. In terms of these divisors, the class of the spectral cover $S$ in the singular limit will be

$$
[S]=6 \sigma_{1}+36 H .
$$

We might hope to get some information about the F-theory geometry from the spectral cover alone. Naively, we may write the algebraic formula for the spectral cover whose class is given in Eq. (6.3) as

$$
S=f_{36} z^{3}+f_{30} x z+f_{27} y,
$$


where $f_{i}$ are generic polynomials of degree $i$ over $\mathbb{P}^{2}$. A generic deformation of the spectral cover of the form (6.4) can be obtained by counting the d.o.f. in the polynomials $f_{36}, f_{30}, f_{27}$, which contain 703,496 , and 406 parameters, respectively. Immediately we see that these numbers are much higher than the dimension of the vector bundle moduli space in our example, which is 292 dimensional. Thus, we can see that the FM transform of the monad in Eq. (3.4) is certainly not a generic spectral cover. This is not too surprising. We saw examples of the spectral cover of monads in Sec. V, and there it was clear that the polynomials are not generic; rather they are dictated by the monad's map (see also [45,49]). In principle, a similar story happens in the current case. We expect that the spectral cover may also be nonreduced/reducible [45]. However, regardless of its explicit form, the question arises, why is the spectral cover forbidden from assuming a generic form? That is, given an explicit starting point [in which the polynomials are determined by the monad map as in Eq. (3.4)], why is the deformation space restricted?

We expect that the answer to this lies with the other half of the spectral data of this monad, that is, the rank 1 sheaf [12] supported over the spectral cover in Eqs. (6.3) and (6.4). It has been observed previously [55] that the Picard group of $S$ may "jump" at higher codimensional loci in moduli space-i.e., so-called Noether-Lefschetz loci. This phenomenon could "freeze" the moduli of the spectral cover to a subspace compatible with the form of the monad map (see also [56]). In terms of the four-dimensional, $\mathcal{N}=1$ EFT, the reduction in the apparent number of singlets (i.e., the nongeneric form of the spectral cover) is a symptom of existence of a specific superpotentialarising from the Gukov-Vafa-Witten form [57]:

$$
W \sim \int_{X} H \wedge \Omega
$$

where $H \sim d B+\omega_{3}^{\mathrm{YM}}-\omega_{3}^{\text {Lorentz }}, \omega_{3}=F \wedge A-\frac{1}{3} A \wedge A \wedge A$ is the Chern-Simons 3-form (and the associated Lorentz quantity built from the spin connection in $\omega_{3}^{\text {Lorentz }}$, and $\Omega$ is the holomorphic $(0,3)$-form on $X$. The existence of this superpotential arises from the presence of the gauge bundle (rather than from quantized flux) (see $[55,58,59]$ for related discussions) but nonetheless stabilizes vector bundle moduli.

As a result, in the dual F-theory EFT, we also expect the existence of a superpotential. Geometrically, since the spectral cover determines part of the complex structure moduli of the Calabi-Yau fourfold, it is clear that the dual of the bundle data given in Eq. (3.4) should include a specific G-flux that stabilizes the moduli through the Gukov-VafaWitten superpotential. It should be noted that there is another way to see the requirement for this flux: Since there are no $D 3$-branes in the F-theory dual [since we have chosen $c_{2}(X)=c_{2}(V)$ in the heterotic theory], G-flux is also necessary for anomaly cancellation.

We have not yet explicitly calculated the FM transform of the heterotic bundle or determined the dual F-theory geometry. The arguments above show that whatever the F-theory geometry, G-flux must play a prominent role, and therefore it cannot be ignored. A similar set of arguments can also be made about the F-theory dual of the heterotic TSD geometry $(\tilde{X}, \tilde{V})$. In this case as well, the naive deformations of the spectral cover are much larger than the dimension of the vector bundle moduli space, and therefore we conclude that Noether-Lefschetz loci/G-flux should be in play.

Despite the fact that flux must be involved in the putative F-theory duality, it still remains to be asked whether the dual F-theory fourfolds might still exhibit multiple fibration structure. That is, could the geometric scenario described in the Introduction with these compatible elliptic/ $/ \mathbb{P}^{1}$ (and hence $K 3$ ) fibrations exist?

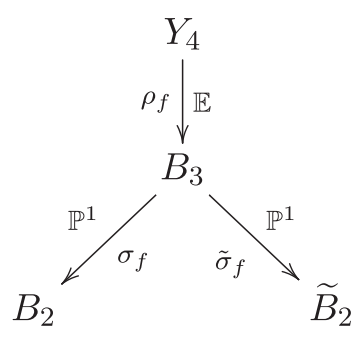

On this front, once again, we see that we must quickly leave behind the standard geometry of heterotic/F-theory duality. As reviewed in Sec. IV, if the heterotic CY threefold is in Weierstrass form, the construction of [12] generates a threefold base $B_{3}$ [see Eq. (4.2)] for the CY fourfold that is a $\mathbb{P}^{1}$ bundle over the base $B_{2}$ (which is the base of the dual elliptically fibered $\mathrm{CY}$ threefold and $K 3$-fibered fourfold). The topology of this bundle (i.e., $B_{3}$ itself) is determined by the second Chern class of the heterotic bundle $c_{2}(V)$. In this context then, we can ask whether or not such a base could admit two different descriptions as a $\mathbb{P}^{1}$ bundle. While multiply fibered $\mathbb{P}^{1}$ bundles certainly exist (for example, the "generalized Hirzebruch" toric threefold defined as the zero twist over $\mathbb{F}_{n}$ or the $n$ twist over $\left.\mathbb{F}_{0}[27,60]\right)$, it is easy to demonstrate that

$$
h^{1,1}\left(B_{3}\right)=1+h^{1,1}\left(B_{2}\right)
$$

for any $\mathbb{P}^{1}$ bundle. As a result, it is clear that there exists no multiply fibered $\mathbb{P}^{1}$ bundles compatible with the $B_{2}$ and $\tilde{B}_{2}$ arising in Sec. III since for those manifolds $B_{2}=\mathbb{P}^{2}$ and $\tilde{B}_{2}=d P_{1}$. Hence $h^{1,1}\left(B_{2}\right)<h^{1,1}\left(\tilde{B}_{2}\right)$ and $h^{1,1}\left(B_{3}\right) \neq$ $h^{1,1}\left(\tilde{B}_{3}\right)$ for threefold bases constructed as $\mathbb{P}^{1}$ bundles.

From the results above, we would be tempted to conclude that the hypothesis we set out to test in the literature (i.e., is heterotic TSD dual to multiply fibered 
geometries in F-theory?) is manifestly false. However, we must first recall that the construction of $K 3$ fibrations in terms of $\mathbb{P}^{1}$-bundle bases $B_{3}$ as commonly used in the literature is not the only possible structure. More general threefold bases $B_{3}$ are possible which are $\mathbb{P}^{1}$ fibrations but not $\mathbb{P}^{1}$ bundles. These fibrations degenerate (as multiple $\mathbb{P}^{1} \mathrm{~s}$ ) over higher codimensional loci in the base $B_{2}$ and are known as "conic bundles" in the literature (see, e.g., [61]).

If we consider this more general class of bases for CY fourfolds, it seems that some possibilities remain. For example, the following threefold,

$$
B_{3}=\left[\begin{array}{l|ll}
\mathbb{P}^{2} & 0 & 1 \\
\mathbb{P}^{1} & 1 & 0 \\
\mathbb{P}^{2} & 1 & 1
\end{array}\right],
$$

is manifestly fibered over both $\mathbb{P}^{2}$ and $d P_{1}$, as required. However, it is unclear that the generic twist of such a fibration is compatible with the topology of the bundles defined in Sec. III. It is possible to generalize simple constructions like the one above to accommodate more general twists by choosing more general toric ambient spaces. However, in each case, we hit a new problem in that the stable degeneration limits of $\mathbb{P}^{1}$ bundles such as that in Eq. (6.7) are not yet understood in the literature (though we are considering such geometries in separate work [14]). As a result, it is a nontrivial task to determine whether such a geometry might arise in the F-theory duals of the examples outlined in Sec. III. To check this, we need precise spectral data. But as explained before, finding the Fourier-Mukai transforms of the heterotic bundles, while possible in principle, is beyond our current computational limits for the bundles in Sec. III.

For now, though, we can conclude that whatever the F-theory correspondence induced from $(0,2)$ target space duality may be, it must expand the current understanding of heterotic/F-theory duality both via the crucial inclusion of G-fluxes (possibly including limits and T-brane solutions) and via more general geometry-in particular, $K 3 / \mathbb{P}^{1}$ fibrations - than has previously been considered.

\section{CONCLUSIONS AND FUTURE DIRECTIONS}

In this work, we have taken a first step toward exploring the consequences of $(0,2)$ target space duality for heterotic/ F-theory duality. In an important proof of principle, we have illustrated that heterotic TSD pairs exist in which both halves of the geometry exhibit Calabi-Yau threefolds with elliptic fibrations. As a result, it is clear that some F-theory correspondence should be induced in these cases. We take several steps to explore the properties of this putative duality. First, we consider the conjecture made previously in the literature that the F-theory realization of TSD could be multiple $K 3$ fibrations of the same elliptically fibered
Calabi-Yau fourfold background of F-theory. To explore this possibility in earnest, we begin in six-dimensional compactifications of heterotic string theory/F-theory and demonstrate that, in general, multiple fibrations within Ftheory CY backgrounds cannot correspond to the (topologically trivial) TSD realizable for bundles on $K 3$ surfaces. Finally, we provide a sketch of the open questions that arise when attempting to directly compute the F-theory duals of four-dimensional heterotic TSD geometries. In particular, we demonstrate that multiple $K 3$ fibrations in F-theory cannot account for $(0,2)$ TSD in the case in which the threefold base $B_{3}$ of the F-theory elliptic fibration takes the form normally assumed - that of a $\mathbb{P}^{1}$ bundle over a (complex) two-dimensional surface $B_{2}$.

There are a number of future directions that naturally lead on from this study, most importantly to explicitly determine the F-theory mechanism that generates dual theories from potentially disparate fourfold geometries. We hope to understand this correspondence in future work. This study has shed light on these questions, however, and has highlighted areas where the current state of the art in the literature is insufficient to determine the dual heterotic/ F-theory geometries.

As noted in Sec. VI, it is clear that new tools will be needed to fully determine this duality. The new geometric features that must be understood in heterotic/F-theory duality in this context clearly extend beyond the canonical assumptions made in [12], and new tools must be developed. These include the following open problems in heterotic/F-theory duality:

(a) Heterotic compactifications on elliptic threefolds with higher rank Mordell-Weil group (as in the examples in Sec. III).

(b) F-theory compactifications on threefold bases that are $\mathbb{P}^{1}$ fibered, but not $\mathbb{P}^{1}$ bundles, i.e., F-theory on elliptic fibrations with conic bundle (see, e.g., [61]) bases.

(c) F-theory duals of degenerate (i.e., nonreduced and reducible) heterotic spectral covers. These seem to be a ubiquitous feature in the context of $(0,2)$ target space duality since the spectral data of monad bundles appear to be generically singular [45].

(d) Four-dimensional T-brane solutions of F-theory (expected to arise in the context of degenerate spectral covers above [16,17,62]).

This last point seems to be an essential part of the story for four-dimensional heterotic/F-theory pairs since degenerate spectral data naturally arise for monad bundles [and hence geometries arising from $(0,2)$ GLSMs]. Moreover, the arguments in Sec. VI make it clear that the d.o.f. of an expected dual F-theory fourfold must be constrained by flux in order to match the moduli count of the heterotic theory. Several of these "missing ingredients" are currently being studied (see [13] for generalizations of heterotic geometries in heterotic/F-theory duality, and [14] for a study of F-theory on conic bundles). We hope that this 
work illustrates the need for these new tools and demonstrates that there remain many interesting open questions within the context of four-dimensional heterotic/F-theory duality. We will return to these open questions in future work.

\section{ACKNOWLEDGMENTS}

The authors would like to thank James Gray, Paul Oehlmann, and Nikhil Raghuram for the useful discussions. In addition, L. B. A. and M. K. gratefully acknowledge the hospitality of the Simons Center for Geometry and Physics (and the semester-long program "The Geometry and Physics of Hitchin Systems") during the completion of this work. The work of L. B. A. is supported by NSF Grant No. PHY1720321.

\section{APPENDIX A: FURTHER EXAMPLES}

In this Appendix, we present some of the exotic cases that we encountered during the search for finding "good examples" of stable, smooth vector bundles over bases that are Weierstrass elliptic fibrations. All of these examples pass the usual necessary conditions for stability such as $h^{0}(V)=0$ and Bogomolov topological constraint, but either the spectrum charged hypermultiplets of the $4 \mathrm{D}$ effective theories are different or the total moduli is not conserved. By using careful Fourier-Mukai analysis, we can show that the first example is indeed unstable, so it explains the discrepancy, but the other two are perfectly stable vector bundles, and we are unable to explain the reason. In the third example in which the spectra match on both sides, one may suggest that the existence of the flux (which must exist due to the generically nonreduced spectral cover) may stabilize the moduli space,

\begin{tabular}{|ccccccc|c||rcc|cc|cc|}
\hline \multicolumn{8}{|c|}{$x_{i}$} & \multicolumn{1}{|c|}{$\Gamma^{j}$} & \multicolumn{1}{|c|}{$\Lambda^{a}$} & \multicolumn{2}{|c|}{$p_{l}$} \\
\hline 3 & 2 & 1 & 0 & 0 & 0 & 0 & -6 & 1 & 0 & 1 & 1 & 1 & -1 & -3 \\
0 & 0 & -2 & 1 & 1 & 0 & 0 & 0 & 1 & 3 & -2 & 2 & 1 & -1 & -4 \\
0 & 0 & -2 & 0 & 0 & 1 & 1 & 0 & 0 & 3 & -2 & 3 & 0 & -1 & -3 \\
\hline
\end{tabular}

with the second Chern classes as

$$
\begin{aligned}
& c_{2}(X)=11 \sigma^{2}+2 \sigma D_{1}+2 \sigma D_{2}-3 D_{1}^{2}-4 D_{1} D_{2}-3 D_{2}^{2}=24 \sigma D_{1}+24 \sigma D_{2}-4 D_{1} D_{2}, \\
& c_{2}(V)=3 \sigma^{2}+11 \sigma D_{1}+9 \sigma D_{2}-D_{1}^{2}-6 D_{1} D_{2}-6 D_{2}^{2}=17 \sigma D_{1}+15 \sigma D_{2}-6 D_{1} D_{2},
\end{aligned}
$$

where $\sigma, D_{1}$, and $D_{2}$ are the section and base divisors, respectively, with $D_{1}^{2}=D_{2}^{2}=0$, and $D_{1} D_{2}=f$ being the class of the generic fiber $f$. The anomaly cancellation is not satisfied in the strong sense, but we can still make sense of it at least as heterotic string theory (maybe not GLSM, but well defined as heterotic string theory). Again, we embed this GLSM in a larger one,

\begin{tabular}{|ccccccccc||ccc||ccccc|cc|}
\hline \multicolumn{8}{c|}{$x_{i}$} & \multicolumn{4}{|c||}{$\Gamma^{j}$} & \multicolumn{4}{|c||}{$\Lambda^{a}$} & \multicolumn{3}{|c|}{$p_{l}$} \\
\hline 3 & 2 & 1 & 0 & 0 & 0 & 0 & 1 & 0 & -6 & -1 & 0 & 1 & 0 & 1 & 1 & 1 & -1 & -3 \\
0 & 0 & -2 & 1 & 1 & 0 & 0 & -3 & 1 & 0 & 3 & -1 & 1 & 3 & -2 & 2 & 1 & -1 & -4 \\
0 & 0 & -2 & 0 & 0 & 1 & 1 & -2 & 1 & 0 & 2 & -1 & 0 & 3 & -2 & 3 & 0 & -1 & -3 \\
\hline
\end{tabular}

with the degrees of the monad maps being as follows:

\begin{tabular}{|ccccc|ccccc|}
\hline \multicolumn{5}{c}{$F^{1}$} & \multicolumn{5}{c|}{$F^{2}$} \\
\cline { 1 - 7 } 0 & 1 & 0 & 0 & 0 & 2 & 3 & 2 & 2 & 2 \\
0 & -2 & 3 & -1 & 0 & 3 & 1 & 6 & 2 & 3 \\
1 & -2 & 3 & -2 & 1 & 3 & 0 & 5 & 0 & 3 \\
\hline
\end{tabular}

After exchanging $\Gamma^{2}, \Gamma^{3}$ (degrees $\left\|G_{2}\right\|,\left\|G_{3}\right\|$, respectively) with $F_{1}^{1}, F_{2}^{1}$, respectively, and integrating out the repeated entries, the dual $(\tilde{X}, \tilde{V})$ can be written as follows: 


\begin{tabular}{|ccccccc||cc||ccccc|cc|}
\hline \multicolumn{8}{c|}{$x_{i}$} & \multicolumn{1}{|c||}{$\Gamma^{j}$} & \multicolumn{1}{|c|}{$\Lambda^{a}$} & \multicolumn{2}{|c|}{$p_{l}$} \\
\hline 3 & 2 & 1 & 0 & 0 & 0 & 0 & -6 & 1 & 0 & 1 & 1 & 1 & -1 & -3 \\
0 & 0 & -3 & 1 & 1 & 1 & 0 & 0 & 0 & 4 & -2 & 2 & 1 & -1 & -4 \\
0 & 0 & -2 & 0 & 0 & 1 & 1 & 0 & 0 & 3 & -2 & 3 & 0 & -1 & -3 \\
\hline
\end{tabular}

The dual geometry is perfectly smooth and the anomaly cancellation condition can also make sense as before. However, the spectrum of charged scalers are not the same, i.e., $h^{1}(V)=121$ while $h^{1}(\tilde{V})=101$. So there should be a problem. We can argue that this is related to the stability.

After a detailed calculation of the Fourier-Mukai transform of $V$, it becomes clear that $F M^{1}(V)$ is of relative rank 1 and degree 2. On the other hand, $F M^{0}(V)$ is also nonzero with relative rank and degree 1 and -1 . It is well known that the Fourier-Mukai transformation of a sheaf of relative rank and degree $(n, d)$ is complex of relative rank and degree $(d,-n)$. So it is clear from the above data that the restriction of $V$ on a generic elliptic fiber $E$ is roughly of the form $\mathcal{O}_{E}(\sigma) \oplus \mathcal{V}_{2}$, where $\mathcal{V}_{2}$ is a rank 2 irreducible bundle of degree -1 on $E$. Obviously it tells us that the bundle must be unstable because it is unstable on generic fibers [even though it seems $h^{0}(V)=0$ ]. As a sanity check, we can compute the rank of $\pi_{*} V$ and $\pi_{*}\left(V \otimes \mathcal{O}_{X}(\sigma)\right)$, and they are 1 and 3 , respectively. This is consistent because $h^{*}\left(\mathcal{V}_{2}\right)=(0,1) \cdot{ }^{14}$ A similar statement can be made about the TSD setup.

\section{APPENDIX B: HIDDEN ISOMORPHISMS}

In this Appendix, we present an example of a TSD pair in which the geometries $(X, V)$ and $(\tilde{X}, \tilde{V})$ are actually equivalent geometries, even though they are described by different algebraic descriptions (of manifolds and monad bundles). Another interesting feature in this case is that both sides of this "trivial" correspondence are elliptically fibered; however, the base manifolds are two different Hirzebruch surfaces, $\mathbb{F}_{0}$ and $\mathbb{F}_{2}$. These base surfaces are distinct as complex manifolds but identical as real (and the elliptic CY threefold over these different surfaces is the same complex manifold). This demonstrates that even trivial TSD correspondences may involve interesting geometric structures.

In the following example, the bundle $\tilde{V}$ on $\tilde{X}$ is a nontrivial rewriting of bundle $V$ on $X$. Both of the CY threefolds are weighted projective space $\mathbb{P}^{2}[123]$ fibered Calabi-Yau threefolds. For $X$, the base is Hirzebruch surface $\mathbb{F}_{0}$, i.e., $B_{2}=\mathbb{P}^{3}[2]$, while for $\tilde{X}$ the base is

$$
\widetilde{B_{2}}=\left[\begin{array}{l|ll}
\mathbb{P}^{3} & 1 & 1 \\
\mathbb{P}^{1} & 1 & 1
\end{array}\right]
$$

which is generically $\mathbb{F}_{0}$, but at special complex structure moduli it jumps to become $\mathbb{F}_{2}$ [21]. A $(0,2)$ target space map can be found that takes $X$ to $\tilde{X}$ [this can be achieved by adding a $\mathbb{P}^{1}$ to the configuration as usual for a $U(1)$-changing TSD pair]. On this manifold, both a tangent bundle and a nontangent bundle will be studied.

\section{Nontrivial rewriting with tangent bundle}

Let us first consider the case of a deformation of the tangent bundle. The GLSM charge matrix is a general deformation of $\left(X, V=T X+\mathcal{O}^{\oplus 2}\right)$ and can be written as follows:

\begin{tabular}{|ccccccc|cc||ccccccc|cc|}
\hline \multicolumn{9}{|c|}{$x_{i}$} & \multicolumn{1}{|c|}{$\Gamma^{j}$} & \multicolumn{1}{|c|}{$\Lambda^{a}$} & \multicolumn{3}{c|}{$p_{l}$} \\
\hline 3 & 2 & 1 & 0 & 0 & 0 & 0 & -6 & 0 & 3 & 2 & 1 & 0 & 0 & 0 & 0 & -6 & 0 \\
0 & 0 & -2 & 1 & 1 & 1 & 1 & 0 & -2 & 0 & 0 & -2 & 1 & 1 & 1 & 1 & 0 & -2 \\
\hline
\end{tabular}

Following the procedure described in the previous section, we will end up with the new charge matrix of the target space dual $(\tilde{X}, \tilde{V})$ :

\footnotetext{
${ }^{14}$ One can also get the same numbers from semistable bundles with rank 3 and degree zero, so they are just necessary conditions.
} 


\begin{tabular}{|c|c|c|c|c|c|c|c|c|c|c|c|c|c|c|c|c|c|c|c|c|}
\hline \multicolumn{9}{|c|}{$x_{i}$} & \multicolumn{3}{|c|}{$\Gamma^{j}$} & \multicolumn{7}{|c|}{$\Lambda^{a}$} & \multicolumn{2}{|c|}{$p_{l}$} \\
\hline$\overline{3}$ & 2 & 1 & $\overline{0}$ & 0 & $\overline{0}$ & 0 & 0 & 0 & -6 & 0 & 0 & 3 & 2 & 1 & 0 & 0 & 0 & 0 & -6 & 0 \\
\hline 0 & 0 & -2 & 1 & 1 & 1 & 1 & 0 & 0 & 0 & -1 & -1 & 0 & 0 & -2 & 1 & 1 & 0 & 2 & 0 & -2 \\
\hline 0 & 0 & 0 & 0 & 0 & 0 & 0 & 1 & 1 & 0 & -1 & -1 & 0 & 0 & 0 & 0 & 0 & 1 & 0 & 0 & -1 \\
\hline
\end{tabular}

The number of both charged and uncharged geometric moduli of the theories on these two manifolds is the same, which suggests that they are indeed target space dual to each other. Such a d.o.f. counting is given by

$$
\begin{array}{ll}
h^{*}(V)=(0,241,1,0) & h^{1,1}(X)+h^{2,1}(X)+h^{1}\left(\operatorname{End}_{0}(V)\right)=3+243+1074=1320, \\
h^{*}(\tilde{V})=(0,241,1,0) & h^{1,1}(\tilde{X})+h^{2,1}(\tilde{X})+h^{1}\left(\operatorname{End}_{0}(\tilde{V})\right)=3+243+1074=1320 .
\end{array}
$$

\section{a. Calculate twist of $V$ and $\tilde{V}$}

Starting with heterotic theory, without loss of generality, the second Chern class can be split as Eq. (4.3), and the heterotic Bianchi identity will imply further that $\eta$ can be parametrized as Eq. (4.4) with the twist of the theory $T^{\prime}=T$. In order to get the twist in our example, one can first calculate the second Chern class of $V$ and $\tilde{V}$ as

$$
\begin{aligned}
& c_{2}(V)=c_{2}(T X)=11 J_{1}^{2}+2 J_{1} J_{2}-2 J_{2}^{2}, \\
& c_{2}(\tilde{V})=c_{2}(\widetilde{T X})=11 J_{1}^{2}+2 J_{1} J_{2}-3 J_{2}^{2}+2 J_{2} J_{3} .
\end{aligned}
$$

For both $X$ and $\tilde{X}$, the section can be parametrized as $\sigma=J_{1}-2 J_{2}$, and the section satisfies the birational condition $\sigma^{2}=-c_{1}(B) \sigma$. Then, by applying Eqs. (4.3) and (4.4), we get

$$
\eta=24 J_{2}, \quad T=12 J_{2}=6 c_{1}(B)
$$

for both $V$ and $\tilde{V}$. This indicates that if we start from a deformation of the tangent bundle, after target space dual we will at least end up with a TSD bundle over the same manifold that is topologically equivalent.

\section{b. Complex deformation of bundle moduli}

We can further compare $V$ and $\tilde{V}$ by analyzing the deformation of these vector bundles. Consider the difference of $V$ and $\tilde{V}$ defined on $B_{2}$ and $\tilde{B}_{2}$ in the sequence separately. They are

$0 \rightarrow V \rightarrow \mathcal{O}(0,1)^{\oplus 2} \rightarrow \mathcal{O}(0,2) \rightarrow 0$

$0 \rightarrow \tilde{V} \rightarrow \mathcal{O}(0,0,1) \oplus \mathcal{O}(0,2,0) \rightarrow \mathcal{O}(0,2,1) \rightarrow 0$,

where $V$ is the kernel of map with two degree $\|1\|$ polynomial on $B_{2}=\left[\mathbb{P}^{3} \mid 2\right], \tilde{V}$ is kernel of map $F$ with degree $\|0,1\|$ and $\|2,0\|$ on

$$
\widetilde{B_{2}}=\left[\begin{array}{l|ll}
\mathbb{P}^{3} & 1 & 1 \\
\mathbb{P}^{1} & 1 & 1
\end{array}\right] .
$$

However, for $\left(\tilde{B}_{2}, \tilde{V}\right)$, if we first solve the polynomial of degree $\|0,1\|$ and put the constraint on the second map with degree $\|2,0\|$, the second map will exactly reduce to a degree $\|2\|$ polynomial on the manifold $\left[\begin{array}{l|ll}\mathbb{P}^{3} & 1 & 1\end{array}\right]$. So it seems that the bundle moduli in $\left(\tilde{B}_{2}, \tilde{V}\right)$ are transformed to the complex moduli in $\left(B_{2}, V\right)$. Then it would be interesting to ask whether it is possible that the complex structure and bundle moduli exchange in $(X, V)$ and $(\tilde{X}, \tilde{V})$.

Before answering this question, there is an important observation that $\widetilde{B_{2}}$ is generically $\mathbb{F}_{0}$, but at a special point it jumps to become $\mathbb{F}_{2}$. Write down the defining equations for

$$
\widetilde{B_{2}}=\left[\begin{array}{l|ll}
\mathbb{P}^{3} & 1 & 1 \\
\mathbb{P}^{1} & 1 & 1
\end{array}\right]
$$

as

$$
\begin{aligned}
z_{0} w_{0}+z_{1} w_{1} & =0, \\
z_{2} w_{0}+\left(\sum_{i=0}^{2} a_{i} z_{i}+\epsilon z_{3}\right) w_{1} & =0
\end{aligned}
$$

with $\left[z_{0}, z_{1}, z_{2}, z_{3}\right] \in \mathbb{P}^{3}$ and $\left[w_{0}, w_{1}\right] \in \mathbb{P}^{1}$. If $\epsilon \neq 0$, this system defines $\mathbb{F}_{0}$. When $\epsilon=0$, a $\mathbb{P}^{1}$ blows up at $(0,0,0,1) \in \mathbb{P}^{3}$, which makes it become $\mathbb{F}_{2}$. So the question about whether the complex structure and bundle moduli exchange in $\left(B_{2}, V\right)$ and $\left(\tilde{B}_{2}, \tilde{V}\right)$ changes to a question about what happens for the geometric moduli of $\left(\tilde{B}_{2}, \tilde{V}\right)$ when $\tilde{B}_{2}$ becomes $\mathbb{F}_{2}$, and the same for tuning the map of the bundle in the $\left(B_{2}, V\right)$ system.

So in calculating the line bundle cohomology in the new system $\left(\tilde{B}_{2}, \tilde{V}\right)$, we will not only count the dimension of the cohomology group appearing in the sequence but also their polynomial representations and the explicit map. More specifically, we will set $z_{3} \in \mathbb{P}^{3}$ in our calculation to 
zero to deform the $\tilde{B}_{2}$ to $F_{2}$ and see what happens. In this case, the line bundle cohomology are $h^{*}(\mathcal{O}(0,1))=$ $\{2,0,0\}, h^{*}(\mathcal{O}(2,0))=\{9,0,0\}, h^{*}(\mathcal{O}(1,1))=\{12,0,0\}$ with and without turning the base manifold. Furthermore, we can check that the cohomology of bundle $h^{*}\left(\tilde{B}_{2}, \tilde{V}\right)=$ $\{4,5,0\}$ will not be affected by the tuning. On the other hand, we can also tune the complex structure of the map $\left(x_{7}=0\right.$ in $\left.F\right)$ in defining the map of $V$ in the $\left(B_{2}, V\right)$ system. Again, the deformation of the map does not change the bundle valued cohomology $h^{*}\left(B_{2}, V\right)=\{1,2,0\}$.

\section{Nontrivial rewriting with general vector bundle}

Similarly, we can consider another example with the same manifolds but different bundles. Again, we start from the following manifold with charge matrix of the form $(\mathrm{X}, \mathrm{V})$ :

\begin{tabular}{|c|c|c|c|c|c|c|c|c|c|c|c|c|c|c|c|}
\hline & \multicolumn{6}{|c|}{$x_{i}$} & \multicolumn{2}{|c|}{$\Gamma^{j}$} & \multicolumn{5}{|c|}{$\Lambda^{a}$} & \multicolumn{2}{|c|}{$p_{l}$} \\
\hline 3 & 2 & 1 & 0 & 0 & 0 & 0 & & 0 & 4 & 2 & & 0 & 0 & -6 & 0 \\
\hline 0 & 0 & -2 & 1 & 1 & 1 & 1 & 0 & -2 & 0 & -2 & 2 & 2 & 1 & 0 & -3 \\
\hline
\end{tabular}

The second Chern class of $(X, T X)$ and $(X, V)$ is given by

$$
c_{2}(T X)=11 J_{1}^{2}+2 J_{1} J_{2}-2 J_{2}^{2}, \quad c_{2}(V)=8 J_{1}^{2}+4 J_{1} J_{2}-2 J_{2}^{2},
$$

which satisfy the $c_{2}$ condition $c_{2}(V) \leq c_{2}(T X)$. The target space dual of this theory is given by the form of $(\tilde{X}, \tilde{V})$,

\begin{tabular}{|ccccccccc|ccc||ccccc|cc|}
\hline \multicolumn{1}{|c|}{$x_{i}$} & \multicolumn{4}{|c||}{$\Gamma^{j}$} & \multicolumn{4}{|c||}{$\Lambda^{a}$} & \multicolumn{3}{|c|}{$p_{l}$} \\
\hline 3 & 2 & 1 & 0 & 0 & 0 & 0 & 0 & 0 & -6 & 0 & 0 & 4 & 2 & 0 & 0 & 0 & -6 & 0 \\
0 & 0 & -2 & 1 & 1 & 1 & 1 & 0 & 0 & 0 & -1 & -1 & 0 & -2 & 1 & 3 & 1 & 0 & -3 \\
0 & 0 & 0 & 0 & 0 & 0 & 0 & 1 & 1 & 0 & -1 & -1 & 0 & 0 & 1 & 0 & 0 & 0 & -1 \\
\hline
\end{tabular}

with the second Chern class

$$
c_{2}(\widetilde{T X})=11 J_{1}^{2}+2 J_{1} J_{2}-3 J_{2}^{2}+2 J_{2} J_{3}, \quad c_{2}(\tilde{V})=8 J_{1}^{2}+4 J_{1} J_{2}-3 J_{2}^{2}+2 J_{2} J_{3} .
$$

Once again, we get their twists of the base to be the same,

$$
\eta=20 J_{2}, \quad T=8 J_{2},
$$

for both $V$ and $\tilde{V}$. These result indicates that this target space dual is just a kind of rewriting of the origin $(X, V)$.

[1] J. Distler and S. Kachru, Duality of $(0,2)$ string vacua, Nucl. Phys. B442, 64 (1995).

[2] L. B. Anderson and H. Feng, New evidence for $(0,2)$ target space duality, J. Phys. A 50, 064004 (2017).

[3] R. Blumenhagen and T. Rahn, Landscape study of target space duality of $(0,2)$ heterotic string models, J. High Energy Phys. 09 (2011) 098.

[4] R. Blumenhagen, Target space duality for $(0,2)$ compactifications, Nucl. Phys. B513, 573 (1998).

[5] R. Blumenhagen, $(0,2)$ Target space duality, CICYs and reflexive sheaves, Nucl. Phys. B514, 688 (1998).
[6] T. Rahn, Target space dualities of heterotic grand unified theories, Proc. Symp. Pure Math. 85, 423 (2012).

[7] T. M. Chiang, J. Distler, and B. R. Greene, Some features of $(0,2)$ moduli space, Nucl. Phys. B496, 590 (1997).

[8] K. Hori, S. Katz, A. Klemm, R. Pandharipande, R. Thomas, C. Vafa, R. Vakil, and E. Zaslow, Mirror symmetry, Clay Mathematics Monographs Vol. 1 (American Mathematical Society, 2003).

[9] D. R. Morrison and C. Vafa, Compactifications of $F$ theory on Calabi-Yau threefolds. I, Nucl. Phys. B473, 74 (1996). 
[10] C. Vafa, Evidence for $F$ theory, Nucl. Phys. B469, 403 (1996).

[11] M. Bershadsky, K. A. Intriligator, S. Kachru, D. R. Morrison, V. Sadov, and C. Vafa, Geometric singularities and enhanced gauge symmetries, Nucl. Phys. B481, 215 (1996).

[12] R. Friedman, J. Morgan, and E. Witten, Vector bundles and $F$ theory, Commun. Math. Phys. 187, 679 (1997).

[13] L. B. Anderson, X. Gao, and M. Karkheiran, Extending the geometry of heterotic spectral cover constructions, arXiv:1912.00971.

[14] L. B. Anderson, J. Gray, M. Karkheiran, P. Oehlmann, and N. Raghuram, Conic bundles in $F$-theory (to be published).

[15] S. Cecotti, C. Cordova, J. J. Heckman, and C. Vafa, T-branes and monodromy, J. High Energy Phys. 07 (2011) 030.

[16] L. B. Anderson, J. J. Heckman, and S. Katz, T-branes and geometry, J. High Energy Phys. 05 (2014) 080.

[17] L. B. Anderson, J. J. Heckman, S. Katz, and L. P. Schaposnik, $T$-branes at the limits of geometry, J. High Energy Phys. 10 (2017) 058.

[18] P. Candelas, A. M. Dale, C. A. Lutken, and R. Schimmrigk, Complete intersection Calabi-Yau manifolds, Nucl. Phys. B298, 493 (1988).

[19] G. Horrocks and D. Mumford, A rank 2 vector bundle on P4 with 15,000 symmetries, Topology 12, 6 (1973).

[20] E. Witten, Phases of $N=2$ theories in two dimensions, Nucl. Phys. B403, 159 (1993).

[21] T. Hubsch, Calabi-Yau manifolds: A Bestiary for Physicists (World Scientific, Singapore, 1994).

[22] L. B. Anderson, Heterotic and $M$-theory compactifications for string phenomenology, arXiv:0808.3621.

[23] M. B. Green, J. H. Schwarz, and E. Witten, Superstring Theory Vol. 2: 25th Anniversary Edition (Cambridge University Press, Cambridge, England, 2012).

[24] L. B. Anderson and M. Karkheiran, TASI lectures on geometric tools for string compactifications, Proc. Sci., TASI2017 (2018) 013 [arXiv:1804.08792].

[25] L. B. Anderson, X. Gao, J. Gray, and S. J. Lee, Fibrations in CICY threefolds, J. High Energy Phys. 10 (2017) 077.

[26] L. B. Anderson, X. Gao, J. Gray, and S. J. Lee, Tools for CICYs in F-theory, J. High Energy Phys. 11 (2016) 004.

[27] L. B. Anderson, X. Gao, J. Gray, and S. J. Lee, Multiple fibrations in Calabi-Yau geometry and string dualities, J. High Energy Phys. 10 (2016) 105.

[28] P. Deligne, Courbes Elliptiques: Formulaire d'aprs J. Tate, in Modular Functions of One Variable IV, edited by B. J. Birch and W. Kuyk, Lecture Notes in Mathematics Vol. 476 (Springer, Berlin, 1975), p. 53.

[29] N. Nakayama, On Weierstrass Models, in Algebraic Geometry and Commutative Algebra, Vol. II (Kinokuniya, Tokyo, 1987), p. 405.

[30] C. Bartocci, U. Bruzzo, and D. Hernández Ruipérez, Fourier-Mukai and Nahm Transforms in Geometry and Mathematical Physics (Springer, New York, 2009).

[31] D. Huybrechts, Fourier-Mukai Transforms in Algebraic Geometry (Oxford University Press, New York, 2007).

[32] R. Hartshorne, Algebraic Geometry (Springer-Verlag, Berlin, 1977).

[33] C. A. Weibel, An Introduction to Homological Algebra (Cambridge University Press, Cambridge, England, 1994).
[34] R. Friedman, Algebraic Surfaces and Holomorphic Vector Bundles (Springer-Verlag, Berlin, 1998).

[35] M.F. Atiyah, Vector Bundles over an Elliptic Curve (London Mathematical Society, London, 1957).

[36] P. S. Aspinwall, Aspects of the hypermultiplet moduli space in string duality, J. High Energy Phys. 04 (1998) 019.

[37] G. Curio and R. Y. Donagi, Moduli in $N=1$ heterotic/ $F$ theory duality, Nucl. Phys. B518, 603 (1998).

[38] R. Donagi and M. Wijnholt, Model building with $F$-theory, Adv. Theor. Math. Phys. 15, 1237 (2011).

[39] F. Denef, Les Houches lectures on constructing string vacua, Les Houches 87, 483 (2008).

[40] T. Weigand, F-theory, Proc. Sci., TASI2017 (2018) 016 [arXiv:1806.01854].

[41] W. Taylor, TASI lectures on supergravity and string vacua in various dimensions, arXiv:1104.2051.

[42] D. R. Morrison and C. Vafa, Compactifications of $F$ theory on Calabi-Yau threefolds. II, Nucl. Phys. B476, 437 (1996).

[43] M. J. Duff, R. Minasian, and E. Witten, Evidence for heterotic/heterotic duality, Nucl. Phys. B465, 413 (1996).

[44] M. Bershadsky, T. M. Chiang, B. R. Greene, A. Johansen, and C. I. Lazaroiu, F-theory and linear sigma models, Nucl. Phys. B527, 531 (1998).

[45] R. Friedman, J. W. Morgan, and E. Witten, Vector bundles over elliptic fibration, arXiv:alg-geom/9709029v1.

[46] P. S. Aspinwall and R. Y. Donagi, The heterotic string, the tangent bundle, and derived categories, Adv. Theor. Math. Phys. 2, 1041 (1998).

[47] R. Donagi and M. Wijnholt, Gluing branes, I, J. High Energy Phys. 05 (2013) 068.

[48] R. Donagi and M. Wijnholt, Gluing branes II: Flavour physics and string duality, J. High Energy Phys. 05 (2013) 092.

[49] C. Beasley, J. J. Heckman, and C. Vafa, GUTs and exceptional branes in F-theory-I, J. High Energy Phys. 01 (2009) 058.

[50] L. B. Anderson, Spectral covers, integrality conditions, and heterotic/ $F$-theory duality, J. Singul. 15, 1 (2016).

[51] R. Donagi and M. Wijnholt, Higgs bundles and UV completion in $F$-theory, Commun. Math. Phys. 326, 287 (2014).

[52] T. Shioda, Mordell-Weil lattices for higher genus fibration over a curve, in New Trends in Algebraic Geometry, edited by K. Hulek, M. Reid, C. Peters, and F. Catanese, London Mathematical Society Lecture Note Series Vol. 264 (Cambridge University Press, Cambridge, England, 1999), p. 359.

[53] R. Wazir, Arithmetic on elliptic threefolds, Compos. Math. 140, 567 (2004).

[54] J. Distler, B. R. Greene, and D. R. Morrison, Resolving singularities in $(0,2)$ models, Nucl. Phys. B481, 289 (1996).

[55] S. Gukov, C. Vafa, and E. Witten, CFT's from Calabi-Yau four-folds, Nucl. Phys. B584, 69 (2000); Erratum, Nucl. Phys. B608, 477(E) (2001).

[56] R. Blumenhagen, B. Jurke, T. Rahn, and H. Roschy, Cohomology of line bundles: A computational algorithm, J. Math. Phys. (N.Y.) 51, 103525 (2010).

[57] L. B. Anderson, J. Gray, A. Lukas, and B. Ovrut, The atiyah class and complex structure stabilization in heterotic 
Calabi-Yau compactifications, J. High Energy Phys. 10 (2011) 032.

[58] L. B. Anderson, J. Gray, A. Lukas, and B. Ovrut, Stabilizing the complex structure in heterotic Calabi-Yau vacua, J. High Energy Phys. 02 (2011) 088.

[59] P. Berglund and P. Mayr, Heterotic string / $F$ theory duality from mirror symmetry, Adv. Theor. Math. Phys. 2, 1307 (1998).
[60] V. Sarkisov, Birational automorphisms of conic bundles, Izv. Akad. Nauk SSSR, Ser. Mat. 17, 177 (1981).

[61] L. B. Anderson, L. Fredrickson, M. Esole, and L. P. Schaposnik, Singular geometry and Higgs bundles in string theory, SIGMA 14, 037 (2018).

[62] T. Shioda, On elliptic modular surfaces, J. Math. Soc. Jpn. 24, 20 (1972). 